\title{
Structure, energetics, and mechanical stability of Fe-Cu bcc alloys from first-principles calculations
}

\author{
Jefferson Z. Liu, A. van de Walle, G. Ghosh, and M. Asta* \\ Department of Materials Science and Engineering, Northwestern University, 2220 Campus Drive, Evanston, Illinois 60208-3108, USA
}

(Received 8 July 2005; revised manuscript received 22 August 2005; published 26 October 2005)

\begin{abstract}
Atomic volumes, magnetic moments, mixing energies, and the elastic properties of bcc $\mathrm{Fe}_{1-x} \mathrm{Cu}_{x}$ solid solutions are studied by $a b$ initio calculations based on the cluster expansion framework. For the calculation of concentration-dependent elastic moduli in disordered solid solutions, we introduce a generalization of the cluster expansion technique that is designed to handle tensorial quantities in high-symmetry phases. Calculated mixing energies, atomic volumes, and magnetic moments are found to be in good agreement with available measurements for metastable alloys prepared through nonequilibrium processing techniques. Additionally, the predicted variations of the bulk modulus and shear moduli $C_{44}$ and $C^{\prime}$ with respect to copper concentration are calculated for the disordered bcc phase. While the bulk modulus and $C_{44}$ are positive for all concentrations, $C^{\prime}$ is predicted to be positive only for $\mathrm{Cu}$ concentration less than 50 atomic $\%$, and negative otherwise. Our results thus indicate that the mechanical instability of bcc $\mathrm{Cu}$ persists over a wide range of compositions. The implications of the present results are discussed in relation to the observed metastability of bcc $\mathrm{Fe}-\mathrm{Cu}$ alloys, and the strengthening mechanism of nanoscale bcc precipitates in an $\alpha$-Fe matrix.
\end{abstract}

DOI: 10.1103/PhysRevB.72.144109

PACS number(s): 62.20.Dc, 81.30.Bx, 64.60.My

\section{INTRODUCTION}

In commercial steels $\mathrm{Cu}$ is a commonly occurring element, present either as an intentionally added alloying species or as an impurity. In high-strength steels, nanometerscale $\mathrm{Cu}$-rich precipitates are utilized to provide substantial precipitate hardening of the $\alpha$-Fe matrix. While the hardening effect of $\mathrm{Cu}$ precipitates has long been known, ${ }^{1}$ the details of the strengthening mechanism remain a topic under debate (e.g., Refs. 2-4, and references therein). In steels employed for nuclear pressure-vessel applications, $\mathrm{Cu}$ impurities are known to form precipitates under irradiation, and in such applications these precipitates represent a primary source of embrittlement. The phenomenon of irradiationinduced precipitation in $\mathrm{Fe}-\mathrm{Cu}$ has been the subject of numerous studies aimed at elucidating the thermodynamic factors and kinetic mechanisms underlying this important decomposition reaction (a review of much of the work along these lines is given in the recent article by Lopasso et al. ${ }^{5}$ ).

At nanometer-scale sizes $\mathrm{Cu}$ precipitates in $\alpha$-Fe are coherent and form with the bcc crystal structure. With increasing size these bcc precipitates are observed to transform to a close-packed phase in the form of a twinned $9 R$ structure. $^{6-11}$ Detailed measurements based on atom-probe microscopy yield precipitate compositions that include substantial amounts of dissolved Fe in binary $\mathrm{Fe}-\mathrm{Cu}$ alloys, ${ }^{6,12}$ as well as a number of other solute species in commercial multicomponent steels. ${ }^{13,14}$

The pronounced effect of bcc-Cu precipitates on the mechanical properties of $\alpha$-Fe provides strong motivation for characterizing the structural, thermodynamic, and elastic properties of bcc $\mathrm{Fe}-\mathrm{Cu}$ solid solutions. Of specific interest are the thermodynamic properties of mixing, which provide the driving force for precipitate nucleation, and the composition dependencies of the atomic volume and elastic moduli that influence dislocation-precipitate interactions. Experi- mentally, measurements of the properties of $\mathrm{Fe}-\mathrm{Cu}$ alloys are complicated by the extremely limited solubility characterizing the solid-state phase diagram between bcc Fe and fcc $\mathrm{Cu}$ under equilibrium conditions. However, nonequilibrium processing methods, including rapid quenching, vapor deposition, and mechanical alloying, have proven highly effective in synthesizing metastable single-phase solid solutions, the properties of which have been investigated extensively. ${ }^{15-27}$ Such detailed experimental work has provided the motivation for a number of theoretical investigations over the past decade that have employed both first-principles and classical-potential methods in studies of the structural, energetic, and magnetic properties of metastable fcc and bcc Fe-Cu alloys. ${ }^{5,28-33}$

While the properties of $\mathrm{Fe}-\mathrm{Cu}$ solid solutions have been the topic of several theoretical investigations, to date little attention has been given to the result that bulk elemental $\mathrm{Cu}$ in a bcc structure is mechanically unstable. Specifically, firstprinciples calculations have predicted that the tetragonal shear modulus $C^{\prime}=\left(C_{11}-C_{12}\right) / 2$ for bcc $\mathrm{Cu}$ is negative (e.g., Refs. 34-37). This result implies that at low temperatures bcc $\mathrm{Cu}$ is unstable with respect to a Bain distortion leading to spontaneous transformation to the equilibrium fcc structure. A detailed understanding of the elastic and thermodynamic properties of bcc $\mathrm{Fe}-\mathrm{Cu}$ alloys requires knowledge of the extent of this instability with respect to both composition and temperature. As discussed further below, such information is particularly valuable in understanding whether the observation of coherent bcc $\mathrm{Cu}$-based precipitates in $\alpha$-Fe arises from a compositional stabilization of this structure, or whether the bcc phase is stabilized by interfacial forces as discussed by Kraft et al. ${ }^{34}$ in relation to the observed stability of bcc $\mathrm{Cu}$ in epitaxial thin films ${ }^{38,39}$ (see below). While the structural stability of elemental systems and ordered compounds has been the subject of many detailed first-principles calculations, far less work has been devoted to alloys, ${ }^{40-43}$ 
due to the inherent challenges associated with the modeling of disordered materials from first principles.

In the present work we investigate the compositiondependent structural, thermodynamic, magnetic, and elastic properties of bcc $\mathrm{Fe}-\mathrm{Cu}$ solid solutions employing a firstprinciples method based on the cluster-expansion framework. ${ }^{44,45}$ This work includes the introduction of a general and computationally efficient method to determine the elastic constant tensor of disordered solid solutions from a set of $a b$ initio calculations for ordered structures. The method represents a generalization of the cluster-expansion framework that is widely employed for the prediction of scalar-valued properties, such as energy and volume, for compositionally disordered alloys. The results of the current study show good agreement with experimental measurements for the composition dependencies of the atomic volume, the enthalpy of mixing and magnetic moment. The tetragonal shear modulus $C^{\prime}$ is calculated to have a highly nonlinear composition dependence, and the mechanical instability of bcc $\mathrm{Cu}$ is predicted to persist up to relatively high Fe concentrations.

In the next section the details of the present firstprinciples approach are given, and our method for modeling the elastic constants of solid solutions is described in detail. In Sec. III we present the results of the calculations for atomic volume, mixing energy, magnetic moment, and elastic moduli, and compare with both previous calculations and available experimental measurements. In Sec. IV we provide a discussion of these results as they relate to the extent of the thermodynamic stability of bcc Fe-Cu alloys, and the mechanism of precipitation strengthening. Section $\mathrm{V}$ provides a brief summary of the work.

\section{FORMALISM}

\section{A. Cluster expansion}

The cluster expansion is a generalization of the wellknown Ising Hamiltonian that is aimed at modeling the energetics of substitutional alloys. In this method, a spin-like occupation variable $\sigma_{i}$ is assigned to every site on the parent lattice (bcc in this paper). Different values of $\sigma_{i}$ represent different atomic species. A particular arrangement of atoms on the lattice is called a configuration and can be represented by a vector $\boldsymbol{\sigma}=\left(\sigma_{1}, \sigma_{2}, \ldots, \sigma_{N}\right)$, which is composed of the $N$ occupation variables for each lattice site. Any configurationdependent physical quantity $F(\boldsymbol{\sigma})$ can be written as a weighted sum of multisite cluster functions $\Phi_{\alpha}(\boldsymbol{\sigma})$ :

$$
F(\boldsymbol{\sigma})=\sum_{\alpha} F_{\alpha} \Phi_{\alpha}(\boldsymbol{\sigma})
$$

because the multisite cluster functions $\Phi_{\alpha}(\boldsymbol{\sigma})$ form a complete basis in the configurational space. ${ }^{44}$ Each cluster is composed of a group of lattice sites $\alpha=\left(p_{1}, p_{2}, \ldots, p_{n_{\alpha}}\right)$, and for the case of a binary alloy the cluster functions take the form of a product of occupation variables over the sites in the cluster:

$$
\Phi_{\alpha}(\boldsymbol{\sigma})=\sigma_{p_{1}} \sigma_{p_{2}} \cdots \sigma_{p_{n_{\alpha}}}
$$

The coefficients $F_{\alpha}$, referred to as effective-cluster interactions (ECIs), embody the information regarding the configurational dependence of property $F(\boldsymbol{\sigma})$. The expression given in Eq. (1) is formally exact if all possible clusters are included. For many applications, good accuracy can be obtained by retaining terms corresponding to only a relatively few pairs, three-body, and four-body clusters. To determine the ECIs, we will make use of the so-called structure inversion method (SIM), ${ }^{46,47}$ in which the ECIs are treated as fitting parameters, and are obtained by fitting the truncated form of Eq. (1) to a set of $a b$ initio calculations for selected ordered configurations on the bcc lattice.

In the present work, cluster expansions will be performed for two types of physical quantities, namely scalar quantities (mixing energy, atomic volume, and magnetic moment) and tensors (elastic constants). In a cluster expansion of a scalar quantity, the ECIs $F_{\alpha}$ in Eq. (1) possess the same symmetry as the parent lattice. That means symmetry-equivalent clusters have the same value of $F_{\alpha}$ and can be grouped as a so-called orbit, $\Omega_{\alpha}$, representing the set of symmetryequivalent clusters. ${ }^{48}$ Using this symmetry argument, we can simplify Eq. (1) as follows:

$$
\frac{F(\boldsymbol{\sigma})}{N}=\sum_{\Omega_{\alpha}} F_{\alpha} m_{\alpha} \hat{\Phi}_{\alpha}(\boldsymbol{\sigma}),
$$

where $\hat{\Phi}_{\alpha}$ represents an average over symmetry-equivalent clusters:

$$
\hat{\Phi}_{\alpha}(\boldsymbol{\sigma})=\frac{1}{N_{\alpha}} \sum_{\beta \in \Omega_{\alpha}} \Phi_{\beta}(\boldsymbol{\sigma}),
$$

with $N_{\alpha}$ the number of the symmetry-equivalent clusters in orbit $\Omega_{\alpha}$ in the whole system and $m_{\alpha}=N_{\alpha} / N$ is the number of clusters of type $\alpha$ per lattice site.

We turn now to the application of the cluster expansion as a framework for computing elastic constants of disordered solid solutions. Since for the solid solution phase the elastic constants share the symmetry of the lattice (cubic in the present case), there exists a simple way to extend the conventional cluster expansion formalism to handle the representation of tensor quantities in this context. The main conceptual issue to resolve is that the elastic constants of the ordered structures used in the structure-inversion method to construct the cluster expansion typically have a lower symmetry than the disordered state; elastic constants that are necessarily identical in the disordered state may thus be different in an ordered structure and it is not clear which one should be used in the fit of the cluster expansion. The idea in the current approach is to symmetrize the tensor associated with each structure by averaging the elastic constant tensor $\mathbf{C}$ transformed under all the symmetry operations $S$ in the point group of the underlying lattice: 


$$
\overline{\mathbf{C}}=\left(\sum_{S} S(\mathbf{C})\right) /\left(\sum_{S} 1\right),
$$

where the $S(\mathbf{C})$ denotes the transformation of $\mathbf{C}$ under the symmetry operation $S$. Specifically, since the elastic constants are a fourth-rank tensor tensor we have

$$
[S(\mathbf{C})]_{i j k l}=s_{i i^{\prime}} s_{j j^{\prime}} s_{k k^{\prime}} s_{l l^{\prime}} C_{i^{\prime} j^{\prime} k^{\prime} l^{\prime}}
$$

using the repeated indices notation and letting $s_{i j}$ denote the matrix representing the symmetry operation $S$. By construction, the symmetrized elastic tensor $(\overline{\mathbf{C}})$ will have the symmetry of the disordered phase regardless of the symmetry of the original tensor (e.g., for an ordered structure). In particular, any elastic constant that must be zero by symmetry in the disordered phase will also be zero in the symmetrized tensor. The symmetrization process can be viewed as creating a mixture of every possible orientation of a given ordered structure that can be obtained by the symmetry operations of the lattice. This picture also helps to motivate the applicability of the cluster expansion formalism since it is clear that the mixtures of various configurations can be used as input data for a cluster expansion fit, by the linearity of the cluster expansion in the $\Phi_{\alpha}(\boldsymbol{\sigma})$.

A cluster expansion can be separately constructed for each of the elements in the symmetrized elastic tensor, i.e., treating each symmetrized modulus as if it were an independent scalar quantity. This is possible because each symmetrized modulus is invariant under any of the symmetry operations of the point group, just like any scalar quantity would be. Of course, a true scalar would also be invariant under any change of coordinates, but this distinction does not affect the construction of the cluster expansion, which only relies on invariance under lattice symmetry operations.

In the case of cubic symmetry, as considered in this work, the symmetrization of the elastic-constant tensor simply amounts to averaging $C^{\prime}=\left(C_{11}-C_{12}\right) / 2$ and $C_{44}$ along the three perpendicular bcc crystal axes, respectively. These two averaged moduli $\overline{C^{\prime}}$ and $\bar{C}_{44}$, together with the bulk modulus $B$, entirely determine the cubic-symmetrized elastic tensor. For configurations with cubic symmetry, such as bcc $\mathrm{Fe}, \mathrm{Cu}$, $\mathrm{B} 2-\mathrm{FeCu}$, a disordered $\mathrm{Fe}-\mathrm{Cu}$ solid solution, and so on, this averaged cubic symmetry elastic tensor is simply equal to the actual elastic tensor.

It is a central issue to determine the number and the type of the clusters used in a cluster expansion. If too few terms are used in the truncated cluster expansion, Eq. (3), the accuracy is limited. If too many terms are kept, the problem of overfitting may manifest itself. The mean-square error of the fit may be very small, but the true predictive power of the cluster expansion for data not included in the fit is much lower. The choice of the clusters should lead to the best compromise between these two undesirable effects. Following Ref. 49, the cross-validation (CV) score is used to evaluate the predictive power of a cluster expansion, which is defined as

$$
(\mathrm{CV})^{2}=\frac{1}{n} \sum_{i=1}^{n}\left(F_{i}-\hat{F}_{i}\right)^{2},
$$

where $F_{i}$ is the calculated physical quantity of structure $i$, while $\hat{F}_{i}$ is the predicted value for structure $i$ obtained from the least square fit to the $(n-1)$ other structures. As the number of the clusters increases, the CV score typically decreases before increasing. A well-defined optimal selection of clusters can thus be obtained by minimizing the CV score. Once this set is chosen, the CV score provides an estimate of the predictive error of the resulting cluster expansion.

In this paper, the cluster expansions are generated using the MIT Ab-initio Phase Stability code (MAPS). ${ }^{49}$ The MAPS code automates the selection of clusters used in the SIM fitting to minimize the $\mathrm{CV}$ score, as well as the selection of the ordered structures used for the fit. The basic idea of the structure-selection algorithm, which is described in detail in Ref. 49, is that each new structure to be included in the fit is as different (in terms of correlations) as possible from the other structures already included in the fit. The algorithm was used initially in the development of the cluster expansion for the energy, and led to the generation of the 28 ordered structures described in Table I. These same structures were also used to formulate the cluster expansions for (in addition to energy) the volume and bulk modulus; for the magnetic moment two additional structures were also included. For the symmetrized elastic moduli $\bar{C}^{\prime}$ and $\bar{C}_{44}$, where the computations were more costly, a subset of roughly $2 / 3$ of the initial 28 alloy structures was used in the generation of the cluster expansion; the final number of structures was chosen based on the value of the $\mathrm{CV}$ score resulting from the fit (further details are given in the following section).

The use of the same set of structures to generate cluster expansions of different quantities can be justified as follows. The structure-generation algorithm used in this work is based on the idea of minimization of the variance of the coefficients in a least-squares fit. It turns out that, in a least-squares regression of some variable $y$ on a vector of explanatory variables $\mathbf{x}$, the variance of the regression coefficients is only a function of (i) the prediction error and (ii) the distribution of $\mathbf{x}$. In the large-sample limit, it is well established that the selection of structures has no effect on (i) but does have an impact on (ii). Hence, an asymptotically valid structure selection rule can be based on the values of $\mathbf{x}$ only. In this application $\mathbf{x}$ is a vector of correlations while $y$ is either energy, volume, magnetic moment, or the elastic constants. Hence, the structure-selection algorithm does not depend on which quantity is being cluster expanded, but rather depends only on the correlation functions of the structures.

Once the cluster expansion has been determined, the properties of a fully disordered solid solution (with sites occupied randomly) can be predicted from

$$
\frac{F_{\text {random }}}{N}=\sum_{\alpha} F_{\alpha} m_{\alpha}\langle\sigma\rangle^{n_{\alpha}},
$$

where $\langle\sigma\rangle$ is the average spin concentration, related to the actual concentration of the alloy $(x)$ through the relation 
TABLE I. A description of structures used in cluster-expansion fits for magnetic moment, mixing energy, bulk modulus, volume, and the symmetrized elastic moduli $\bar{C}^{\prime}$ and $\bar{C}_{44}$. In the last four columns a + sign designates that the structure was used in the cluster expansion fit for the given property.

\begin{tabular}{|c|c|c|c|c|c|c|c|c|c|c|c|}
\hline \multirow[b]{2}{*}{ Structure ID } & \multirow[b]{2}{*}{ Space group (\#) } & \multirow[b]{2}{*}{ Wyckoff position } & \multirow[b]{2}{*}{$x$} & \multirow[b]{2}{*}{$y$} & \multirow[b]{2}{*}{$z$} & \multirow[b]{2}{*}{$a / b / c(\AA)$} & \multirow[b]{2}{*}{$\alpha / \beta / \gamma$} & \multicolumn{4}{|c|}{ Cluster expansion } \\
\hline & & & & & & & & $M$ & $\left(\Delta E_{\operatorname{mix}}, B, V_{0}\right)$ & $\overline{C^{\prime}}$ & $\bar{C}_{44}$ \\
\hline 0 & $\operatorname{Im} \overline{3} m(229)$ & $2 a(\mathrm{Fe})$ & 0 & 0 & 0 & 2.826 & & + & + & + & + \\
\hline \multirow[t]{3}{*}{10} & Cmmm (65) & $2 a(\mathrm{Cu})$ & 0.000 & 0.000 & 0.000 & 2.891 & & + & + & + & \\
\hline & & $2 d(\mathrm{Fe})$ & 0.000 & 0.000 & 0.500 & 4.089 & & & & & \\
\hline & & $4 l(\mathrm{Fe})$ & 0.500 & 0.000 & 0.254 & 8.178 & & & & & \\
\hline \multirow[t]{3}{*}{16} & Immm (71) & $2 c(\mathrm{Cu})$ & 0.000 & 0.000 & 0.500 & 4.055 & & + & + & + & + \\
\hline & & $2 a(\mathrm{Fe})$ & 0.000 & 0.000 & 0.000 & 2.868 & & & & & \\
\hline & & $4 j(\mathrm{Fe})$ & 0.500 & 0.000 & 0.255 & 8.111 & & & & & \\
\hline \multirow[t]{2}{*}{24} & $R \overline{3} m(166)$ & $3 b(\mathrm{Cu})$ & 0.000 & 0.000 & 0.500 & 6.213 & 100 & + & + & + & \\
\hline & & $9 d(\mathrm{Fe})$ & 0.833 & 0.167 & 0.167 & & & & & & \\
\hline \multirow[t]{3}{*}{26} & $F m \overline{3} m(225)$ & $4 b(\mathrm{Cu})$ & 0.500 & 0.500 & 0.500 & 5.736 & & + & + & & + \\
\hline & & $4 a(\mathrm{Fe})$ & 0.000 & 0.000 & 0.000 & & & & & & \\
\hline & & $8 c(\mathrm{Fe})$ & 0.750 & 0.750 & 0.750 & & & & & & \\
\hline \multirow[t]{4}{*}{211} & $R \overline{3} m(166)$ & $6 c(\mathrm{Cu})$ & 0.000 & 0.000 & 0.857 & 8.497 & 100 & + & + & & \\
\hline & & $3 a(\mathrm{Fe})$ & 0.000 & 0.000 & 0.000 & & & & & & \\
\hline & & $6 c(\mathrm{Fe})$ & 0.000 & 0.000 & 0.287 & & & & & & \\
\hline & & $6 c(\mathrm{Fe})$ & 0.000 & 0.000 & 0.426 & & & & & & \\
\hline \multirow[t]{3}{*}{6} & I4/mmm (139) & $2 b(\mathrm{Cu})$ & 0.000 & 0.000 & 0.500 & 2.879 & & + & & + & + \\
\hline & & $4 e(\mathrm{Fe})$ & 0.000 & 0.000 & 0.174 & 2.879 & & & & & \\
\hline & & & & & & 8.637 & & & & & \\
\hline \multirow[t]{3}{*}{8} & $P \overline{3} m 1$ (164) & $1 b(\mathrm{Cu})$ & 0.000 & 0.000 & 0.500 & 4.034 & 90 & + & + & + & + \\
\hline & & $2 d(\mathrm{Fe})$ & 0.333 & 0.667 & 0.129 & 4.034 & 90 & & & & \\
\hline & & & & & & 2.470 & 120 & & & & \\
\hline \multirow[t]{3}{*}{37} & I4/mmm (139) & $4 e(\mathrm{Cu})$ & 0.000 & 0.000 & 0.800 & 2.874 & & + & + & & \\
\hline & & $2 a(\mathrm{Fe})$ & 0.000 & 0.000 & 0.000 & 2.874 & & & & & \\
\hline & & $4 e(\mathrm{Fe})$ & 0.000 & 0.000 & 0.597 & 14.368 & & & & & \\
\hline \multirow[t]{3}{*}{2} & Cmmm (65) & $2 a(\mathrm{Cu})$ & 0.000 & 0.000 & 0.000 & 2.877 & & + & + & + & + \\
\hline & & $2 c(\mathrm{Fe})$ & 0.500 & 0.000 & 0.500 & 4.068 & & & & & \\
\hline & & & & & & 4.068 & & & & & \\
\hline \multirow[t]{2}{*}{3} & $P m \overline{3} m(221)$ & $1 a(\mathrm{Cu})$ & 0.000 & 0.000 & 0.000 & 2.887 & & + & + & + & + \\
\hline & & $1 b(\mathrm{Fe})$ & 0.500 & 0.500 & 0.500 & & & & & & \\
\hline \multirow[t]{3}{*}{11} & Cmma (67) & $4 g(\mathrm{Cu})$ & 0.500 & 0.750 & 0.875 & 2.872 & & + & + & & + \\
\hline & & $4 g(\mathrm{Fe})$ & 0.500 & 0.750 & 0.380 & 4.062 & & & & & \\
\hline & & & & & & 8.123 & & & & & \\
\hline 14 & $P 4 / n m m(129)$ & $2 c(\mathrm{Cu})$ & 0.750 & 0.750 & 0.874 & 2.880 & & + & + & + & + \\
\hline & & $2 c(\mathrm{Fe})$ & 0.750 & 0.750 & 0.386 & 2.880 & & & & & \\
\hline & & & & & & 5.760 & & & & & \\
\hline 17 & Imma (74) & $4 e(\mathrm{Cu})$ & 0.000 & 0.250 & 0.627 & 4.080 & & + & + & + & + \\
\hline & & $4 e(\mathrm{Fe})$ & 0.000 & 0.250 & 0.115 & 2.885 & & & & & \\
\hline & & & & & & 8.159 & & & & & \\
\hline 27 & $F d \overline{3} m(227)$ & $8 b(\mathrm{Cu})$ & 0.625 & 0.625 & 0.625 & 5.747 & & + & + & + & + \\
\hline & & $8 a(\mathrm{Fe})$ & 0.875 & 0.875 & 0.875 & & & & & & \\
\hline 93 & $\mathrm{Cm}(8)$ & $2 a(\mathrm{Cu})$ & 0.833 & 0.500 & 0.334 & 7.041 & 90 & + & + & & \\
\hline & & $2 a(\mathrm{Cu})$ & 0.167 & 0.500 & 0.167 & 4.065 & & & & & \\
\hline & & $2 a(\mathrm{Cu})$ & 0.000 & 0.000 & 0.999 & 4.979 & & & & & \\
\hline
\end{tabular}


TABLE I. (Continued.)

\begin{tabular}{|c|c|c|c|c|c|c|c|c|c|c|c|}
\hline \multirow[b]{2}{*}{ Structure ID } & \multirow[b]{2}{*}{ Space group (\#) } & \multirow[b]{2}{*}{ Wyckoff position } & \multirow[b]{2}{*}{$x$} & \multirow[b]{2}{*}{$y$} & \multirow[b]{2}{*}{$z$} & \multirow[b]{2}{*}{$a / b / c(\AA)$} & \multirow[b]{2}{*}{$\alpha / \beta / \gamma$} & \multirow[b]{2}{*}{$M$} & \multicolumn{2}{|c|}{ Cluster expansion } & \multirow[b]{2}{*}{$\bar{C}_{44}$} \\
\hline & & & & & & & & & $\left(\Delta E_{\mathrm{mix}}, B, V_{0}\right)$ & $\overline{C^{\prime}}$ & \\
\hline & & $2 a(\mathrm{Fe})$ & 0.833 & 0.500 & 0.667 & & & & & & \\
\hline & & $2 a(\mathrm{Fe})$ & 0.167 & 0.500 & 0.831 & & & & & & \\
\hline & & $2 a(\mathrm{Fe})$ & 0.000 & 0.000 & 0.503 & & & & & & \\
\hline \multirow[t]{4}{*}{116} & $P 4 / m m m(123)$ & $1 c(\mathrm{Cu})$ & 0.500 & 0.500 & 0.000 & 2.880 & & + & + & & \\
\hline & & $2 g(\mathrm{Cu})$ & 0.000 & 0.000 & 0.169 & 2.880 & & & & & \\
\hline & & $1 b(\mathrm{Fe})$ & 0.000 & 0.000 & 0.500 & 8.641 & & & & & \\
\hline & & $2 h(\mathrm{Fe})$ & 0.500 & 0.500 & 0.659 & & & & & & \\
\hline \multirow[t]{3}{*}{39} & I4/mmm (139) & $2 a(\mathrm{Cu})$ & 0.000 & 0.000 & 0.000 & 2.886 & & + & & & \\
\hline & & $4 e(\mathrm{Cu})$ & 0.000 & 0.000 & 0.399 & 2.886 & & & & & \\
\hline & & $4 e(\mathrm{Fe})$ & 0.000 & 0.000 & 0.206 & 14.43 & & & & & \\
\hline \multirow[t]{3}{*}{54} & $R \overline{3} m(166)$ & $3 a(\mathrm{Cu})$ & 0.000 & 0.000 & 0.000 & 6.891 & 100 & + & + & & \\
\hline & & $6 c(\mathrm{Cu})$ & 0.000 & 0.000 & 0.802 & & & & & & \\
\hline & & $6 c(\mathrm{Fe})$ & 0.000 & 0.000 & 0.404 & & & & & & \\
\hline \multirow[t]{3}{*}{7} & I4mm (107) & $2 a(\mathrm{Cu})$ & 0.000 & 0.000 & 0.335 & 2.890 & & + & + & + & + \\
\hline & & $2 a(\mathrm{Cu})$ & 0.000 & 0.000 & 0.998 & 2.890 & & & & & \\
\hline & & $2 a(\mathrm{Fe})$ & 0.000 & 0.000 & 0.667 & 8.670 & & & & & \\
\hline \multirow[t]{3}{*}{9} & $C m(8)$ & $2 a(\mathrm{Cu})$ & 0.167 & 0.000 & 0.704 & 7.070 & & + & + & + & + \\
\hline & & $2 a(\mathrm{Cu})$ & 0.000 & 0.000 & 0.963 & 4.082 & & & & & \\
\hline & & $2 a(\mathrm{Fe})$ & 0.833 & 0.500 & 0.333 & 2.500 & & & & & \\
\hline \multirow[t]{6}{*}{94} & $\mathrm{Cm}(8)$ & $2 a(\mathrm{Cu})$ & 0.833 & 0.500 & 0.335 & 7.056 & & + & + & & \\
\hline & & $2 a(\mathrm{Cu})$ & 0.167 & 0.500 & 0.171 & 4.074 & & & & & \\
\hline & & $2 a(\mathrm{Cu})$ & 0.833 & 0.500 & 0.832 & 4.990 & & & & & \\
\hline & & $2 a(\mathrm{Cu})$ & 0.000 & 0.000 & 0.995 & & & & & & \\
\hline & & $2 a(\mathrm{Fe})$ & 0.167 & 0.500 & 0.669 & & & & & & \\
\hline & & $2 a(\mathrm{Fe})$ & 0.000 & 0.000 & 0.497 & & & & & & \\
\hline \multirow[t]{3}{*}{12} & Cmmm (65) & $2 c(\mathrm{Cu})$ & 0.500 & 0.000 & 0.500 & 2.884 & & + & + & + & \\
\hline & & $4 k(\mathrm{Cu})$ & 0.000 & 0.000 & 0.750 & 4.079 & & & & & \\
\hline & & $2 b(\mathrm{Fe})$ & 0.500 & 0.000 & 0.000 & 8.158 & & & & & \\
\hline \multirow[t]{3}{*}{18} & $\operatorname{Immm}(71)$ & $2 b(\mathrm{Cu})$ & 0.500 & 0.000 & 0.000 & 4.091 & & + & + & + & + \\
\hline & & $4 i(\mathrm{Cu})$ & 0.000 & 0.000 & 0.253 & 2.893 & & & & & \\
\hline & & $2 d(\mathrm{Fe})$ & 0.000 & 0.500 & 0.000 & 8.182 & & & & & \\
\hline 20 & $P 4 / m m m(123)$ & $1 b(\mathrm{Cu})$ & 0.000 & 0.000 & 0.500 & 4.095 & & + & + & + & \\
\hline & & $2 f(\mathrm{Cu})$ & 0.000 & 0.500 & 0.000 & 4.095 & & & & & \\
\hline & & $1 d(\mathrm{Fe})$ & 0.500 & 0.500 & 0.500 & 2.896 & & & & & \\
\hline 23 & $P 2 / m(10)$ & $1 e(\mathrm{Cu})$ & 0.500 & 0.500 & 0.000 & 2.503 & 90 & + & + & & \\
\hline & & $2 m(\mathrm{Cu})$ & 0.733 & 0.000 & 0.750 & 4.087 & 100 & & & & \\
\hline & & $1 f(\mathrm{Fe})$ & 0.000 & 0.500 & 0.500 & 4.792 & 90 & & & & \\
\hline 25 & $R \overline{3} m(166)$ & $9 d(\mathrm{Cu})$ & 0.833 & 0.667 & 0.167 & 6.286 & 100 & + & + & & \\
\hline & & $3 b(\mathrm{Fe})$ & 0.000 & 0.000 & 0.500 & & & & & & \\
\hline 28 & $F m \overline{3} m(225)$ & $4 b(\mathrm{Cu})$ & 0.500 & 0.500 & 0.500 & 5.783 & & + & + & & + \\
\hline & & $8 c(\mathrm{Cu})$ & 0.750 & 0.750 & 0.750 & & & & & & \\
\hline & & $4 a(\mathrm{Fe})$ & 0.000 & 0.000 & 0.000 & & & & & & \\
\hline 56 & $R \overline{3} m(166)$ & $6 c(\mathrm{Cu})$ & 0.000 & 0.000 & 0.901 & 6.903 & 100 & + & + & + & + \\
\hline & & $6 c(\mathrm{Cu})$ & 0.000 & 0.000 & 0.298 & & & & & & \\
\hline & & $3 b(\mathrm{Fe})$ & 0.000 & 0.000 & 0.500 & & & & & & \\
\hline 1 & $\operatorname{Im} \overline{3} m$ (229) & $2 a(\mathrm{Cu})$ & 0.000 & 0.000 & 0.000 & 2.891 & & + & + & + & + \\
\hline
\end{tabular}


$\langle\sigma\rangle=2 x-1$, and $n_{\alpha}$ is the number of sites in cluster $\alpha$. Note that the special form of the cluster functions $\Phi_{\alpha}(\boldsymbol{\sigma})[\mathrm{Eq} .(2)]$ in a random alloy directly follows from the fact that the spins on each site are statistically independent, so that the expectation of a product of spins factors as the product of the expectations of the spins on each site. Alternatively, the properties of a short-range ordered or clustered solid solution can be obtained by substituting the actual multisite spin correlations (obtained, for instance, from a Monte Carlo simulation) into Eq. (3). In the present work we compare the results of cluster-expansion predictions for solid-solution phases with properties measured in alloys processed under highly nonequilibrium conditions, where the extent of (clustering) short-range order is presumably small. A detailed theoretical analysis of the effects of short-range order on the calculated properties would require an accurate prediction of the temperature scale for the $\mathrm{Fe}-\mathrm{Cu}$ miscibility gap, which in turn is expected to require a consideration of vibrational contributions in the calculation of the mixing free energy (see the Discussion). Due to the expected highly anharmonic nature of the vibrational free energy for this system, such calculations lie beyond the scope of the current work and all comparisons with experiments are made under the assumption of random configurational disorder.

\section{B. Ab initio calculations}

The $a b$ initio calculations in this work were carried out using the $a b$ initio total-energy and molecular-dynamics program VASP (Vienna $a b$ initio simulation package). ${ }^{50,51}$ The calculations were carried out with the projector augmented wave (PAW) method, ${ }^{52,53}$ employing the generalized gradient approximation (GGA) formulated by Perdew and Wang. ${ }^{54}$ Electronic wave functions were expanded in plane waves with a kinetic energy cutoff of $410 \mathrm{eV}$, which is about 1.5 times of the VASP-default value for $\mathrm{Cu}$ and $\mathrm{Fe}$. All calculations were performed spin polarized. In calculations of the equations of state (yielding the energy, atomic volume, and bulk modulus), the Brillouin zone was sampled employing Monkhorst-Pack ${ }^{55}$ k-point meshes with roughly constant mesh densities corresponding to a $13 \times 13 \times 13$ grid for the primitive bcc lattice. Convergence tests were conducted for simple structures including bcc Fe, $\mathrm{Cu}$ and $\mathrm{B} 2-\mathrm{FeCu}$, which indicated that with the chosen plane-wave cutoff and k-point density, the calculated energies are converged to within $0.5 \mathrm{meV} /$ atom. For calculations of $C^{\prime}$ and $C_{44}$, we used the same plane-wave cutoff, and slightly higher k-point mesh densities approximating a sampling of $16 \times 16 \times 16$ and $25 \times 25 \times 25$ grids for the primitive bcc structure, respectively. The electronic energy levels were broadened using the Methfessel-Paxton scheme ${ }^{56}$ with a broadening of $0.1 \mathrm{eV}$.

For each ordered structure to be included in the cluster expansion fit, our approach consisted of the following steps. We start with the unrelaxed ordered structure whose lattice vectors are integral linear combinations of the primitive bcc lattice vectors, and whose atomic positions are located at the sites of an ideal bcc lattice. We then consider a range of positive and negative levels of isotropic strain, and for each value of the strain we perform a total energy calculation relaxing only the internal degrees of freedom, while fixing the shape of the unit cell at its ideal bcc geometry. The resulting energy versus volume plot is then fit with a Murnaghan equation of state to obtain the equilibrium energy $\left(E_{0}\right)$, bulk modulus $(B)$, and atomic volume $\left(V_{0}\right)$. These values correspond to a constrained equilibrium since the unitcell shape is forced to maintain its ideal bcc geometry. Nevertheless, this "cubic" geometry is a convenient reference state that can be compared across structures differing in their symmetry.

In the next step, we impose various levels of anisotropic strains to this volume-relaxed cubic reference state and compute energies and/or stresses (see later) while relaxing all internal degrees of freedom. To derive elastic constants from these calculations, we make use of an expansion of the energy as a function of strain $\epsilon_{i j}$ up to second order:

$$
E(\epsilon)=E_{0}+\sigma_{i j} \epsilon_{i j}+\frac{1}{2} C_{i j k l} \epsilon_{i j} \epsilon_{k l} \text {. }
$$

The reference state $\left(\epsilon_{k l}=0\right)$ is one in which only volume and the internal degrees of freedom are relaxed. The quantities $E_{0}, \sigma_{i j}$, and $C_{i j k l}$ are, respectively, the energy, the stress in the solid and the elastic constants in this "cubic" reference state.

Equation (6) makes a direct connection with the method employed by Craievich et al., ${ }^{40,41}$ who apply the cluster expansion formalism to the function $E(\epsilon)$ directly, while we apply it to the coefficients of the Taylor expansion of $E(\epsilon)$ around a high symmetry point. In the current approach, the $\sigma_{i j}$ and $C_{i j k l}$ tensors are symmetrized to be made compatible with the cubic point group of the disordered system. In this symmetrization process, the $\sigma_{i j}$ parameter vanishes and is therefore not considered further. For a cubic parent lattice the elastic constant tensor $C_{i j k l}$ reduces to three independent components, namely the bulk modulus $B$, which was already determined by the volume optimization, the $\overline{C^{\prime}}$ modulus, and the shear modulus $\bar{C}_{44}$. The latter two quantities are determined by applying strains specifically adapted to isolate each modulus, as described in the following.

\section{Tetragonal shear modulus $\overline{C^{\prime}}$}

We start from the "cubic" reference state described above and impose a volume-conserving tetragonal strain characterized by $\epsilon_{33}=-2 \epsilon_{22}=-2 \epsilon_{11} \equiv \epsilon_{z}$, where all strains and stresses are defined in the coordinate system of the reference bcc parent lattice. From Eq. (6) the energy as a function of $\epsilon_{z}$ can be fit to the form

$$
E\left(\epsilon_{z}\right)=E_{0}+\sigma_{z} \epsilon_{z}+\frac{3}{2} C_{z} \epsilon_{z}^{2}
$$

where $\sigma_{z}=\sigma_{33}-\left(\sigma_{11}+\sigma_{22}\right) / 2$ and $C_{z}=\left(C_{1111} / 4+C_{2222} / 4\right.$ $\left.+C_{3333}+C_{1122} / 2-C_{1133}-C_{2233}\right) / 3$. The values of $\sigma_{z}$ and $C_{z}$ are then derived by fitting Eq. (7) to total energies calculated for seven values of $\epsilon_{z}$, with magnitudes chosen to ensure that the quadratic expansion is sufficiently accurate. In a similar manner, values of the analogous variables $\sigma_{x}$ and $C_{x}$ are derived by computing the change in energy due to a strain state $\epsilon_{11}=-2 \epsilon_{22}=-2 \epsilon_{33} \equiv \epsilon_{x}$, and $\sigma_{y}$ and $C_{y}$ from the energy versus strain $\epsilon_{22}=-2 \epsilon_{11}=-2 \epsilon_{33} \equiv \epsilon_{y}$. With 
the resulting fitted values the symmetrized elastic modulus $\overline{C^{\prime}}$ is determined as $\overline{C^{\prime}}=\left(C_{x}+C_{y}+C_{z}\right) / 3$ $=\left[\left(C_{1111}+C_{2222}+C_{3333}\right) / 3-\left(C_{1122}+C_{1133}+C_{2233}\right) / 3\right] / 2$.

From this final expression, it is easy to verify that in the cubic disordered phase $\overline{C^{\prime}}=\left(C_{11}-C_{12}\right) / 2=C^{\prime}$.

\section{Shear modulus $\bar{C}_{44}$}

A procedure similar to that outlined in the previous paragraph can be employed to derive $\bar{C}_{44}$ by calculating the change in energy resulting from the application of shear strains. In practice, such calculations are substantially more computationally demanding than those described for $\overline{C^{\prime}}$ due to the reduction in symmetry that typically results from imposing a shear strain. The issue of computational efficiency is particularly important in the construction of a cluster expansion that generally requires calculations for many ordered structures. In computing $\bar{C}_{44}$ we thus make use of the stress tensor computed within VASP, enabling us to compute elastic moduli from calculations at single, rather than multiple, strain values. Specifically, we consider the change in shear stress $\left(\sigma_{12}\right)$ resulting by imposing a shear strain $\epsilon_{12}$ to the cubic reference state. From Eq. (7), we have

$$
\sigma_{12}\left(\epsilon_{12}\right)=\sigma_{12}\left(\epsilon_{12}=0\right)+2 C_{1212} \epsilon_{12} .
$$

From the values of $\sigma_{12}$ calculated in the reference state $\left(\epsilon_{12}=0\right)$, and with a small imposed strain $\epsilon_{12}$ (with a typical magnitude of 0.01), the modulus $C_{1212}$ can be derived. In a similar way we compute values for $C_{1313}$ and $C_{2323}$, from which we derive the symmetrized modulus $\bar{C}_{44}$ $=\left(C_{1212}+C_{1313}+C_{2323}\right) / 3$. For simple ordered structures with cubic symmetry, including bcc $\mathrm{Fe}$ and $\mathrm{Cu}$ and $\mathrm{B} 2 \mathrm{FeCu}$, we have checked the accuracy of this method, based on calculations of stresses, relative to the more conventional approach for computing elastic moduli involving fitting the energy versus strain; good agreement, at the level of 5\%, was obtained for the two alternative methods.

\section{RESULTS}

In Table II results for atomic volume, energy, and elastic moduli of elemental $\mathrm{Fe}$ and $\mathrm{Cu}$ are compared with previous calculations and experimental measurements. Compared to measurements performed at about $4 \mathrm{~K},{ }^{57,58}$ the atomic volumes for bcc $\mathrm{Fe}$ are computed to be smaller by roughly $3.4 \%$. Our calculated values for the bulk modulus $B$ and $C^{\prime}$ are larger than the low-temperature measured values by roughly $10 \%$ and $30 \%$, respectively, while $C_{44}$ is smaller by about $20 \%$. The present theoretical values for atomic volume and elastic moduli show good agreement (at the level of a few percent) with previous all-electron GGA calculations, suggesting that much of the discrepancy with measurements for bcc Fe is attributable to the GGA. For fcc $\mathrm{Cu}$, the agreement between the present calculations and low-temperature measurements for atomic volume and elastic moduli are found to be slightly better than for Fe. For bcc $\mathrm{Cu}$, where a comparison with experiment is not possible, we compare the results of our current GGA calculations for elastic moduli with available previous calculations based primarily on the local-density approximation (LDA); the GGA results are found to be substantially lower for $B, C_{44}$, and $C^{\prime}$, consistent with the larger atomic volume predicted by the GGA. Note in particular that the current GGA result of $C^{\prime}=-14.5 \mathrm{GPa}$ for bcc $\mathrm{Cu}$ is considerably more negative (by $8.5 \mathrm{GPa}$ ) than the value calculated by LDA in the work of Wang et al.. ${ }^{36,37}$

Table III presents a comparison between our results for atomic volume, bulk modulus, and formation energy $\left(\Delta E_{\text {form }}\right)$, and previously published GGA calculations for ordered bcc-based $\mathrm{Fe}-\mathrm{Cu}$ compounds. Very good overall agreement is found between the current and previous calculations. In Table III, the formation energy for a $\mathrm{Fe}_{1-x} \mathrm{Cu}_{x}$ compound is defined as the difference between the energy of the compound, and the concentration-weighted average of the energies for bcc Fe and fcc $\mathrm{Cu}: \Delta E_{\text {form }}$ $=E_{0}\left(\mathrm{Fe}_{1-x} \mathrm{Cu}_{x}\right)-(1-x) E_{0}(\mathrm{Fe}, \mathrm{bcc})-x E_{0}(\mathrm{Cu}, \mathrm{fcc})$, where $E_{0}(\mathrm{Fe}, \mathrm{bcc})$ and $E_{0}(\mathrm{Cu}, \mathrm{fcc})$ are the energies per atom for bcc $\mathrm{Fe}$ and fcc $\mathrm{Cu}$, respectively.

The details related to the cluster expansion fits for the mixing energy $\left(\Delta E_{m i x}\right)$, the atomic volume $\left(V_{0}\right)$, magnetic moment $(M)$, bulk modulus $(B)$, and the cubic-symmetrized elastic moduli $\left(\overline{C^{\prime}}\right.$ and $\left.\bar{C}_{44}\right)$ are summarized in Table IV. The mixing energy (as opposed to the formation energy, defined above) represents the difference in energy between the alloy and a concentration-weighted average of the energies of elemental bcc $\mathrm{Fe}$ and bcc $\mathrm{Cu}$, i.e., for a compound $\mathrm{Fe}_{1-x} \mathrm{Cu}_{x}$ we have $\Delta E_{\text {mix }}=E_{0}\left(\mathrm{Fe}_{1-x} \mathrm{Cu}_{x}\right)-(1-x) E_{0}(\mathrm{Fe}, \mathrm{bcc})$ $-x E_{0}(\mathrm{Cu}, \mathrm{bcc})$. In the fitting of $\Delta E_{\text {mix }}$, it was found that seven pair and two triplet cluster interactions were required to fit the 28 calculated values with a CV score of $0.013 \mathrm{eV} /$ atom (the maximum fitting error was $0.018 \mathrm{eV} /$ atom). In this mixing-energy cluster expansion, the nearest-neighbor ECI was dominant with a value of $-0.034 \mathrm{eV}$, which is three times larger in magnitude than the second-neighbor pair interaction, and one and two orders of magnitude larger than the more distant pair and triplet interactions, respectively. The quality of the resulting fit can be seen by comparing the open (direct VASP calculations) and filled (cluster-expansion fit) symbols in Fig. 1 that represent results for the 28 ordered structures, described in Table I, generated by the MAPS code for the cluster-expansion fits. Similar comparisons between calculations and the clusterexpansion fits for $V_{0}$, magnetic moment $M, B, \overline{C^{\prime}}$, and $\bar{C}_{44}$ are given in Figs. 2, 3, 4, 5, and 6, respectively.

The mixing-energy results in Fig. 1 are seen to be positive at all concentrations, for all of the ordered structures considered, as well as the random solid solution. These results are consistent with the presence of a metastable miscibility gap for bcc $\mathrm{Fe}-\mathrm{Cu}$ solid solutions in the assessed phase diagram. ${ }^{74}$ In Fig. 7 these energy results are compared with experimental calorimetry measurements for metastable single-phase bcc solid solutions prepared by mechanical alloying, as reported in the studies by Eckert et $a .^{22}$ and Ma et al. ${ }^{23}$ Also shown in Fig. 7 are results of recently published calculations by Gong et al., ${ }^{33}$ obtained from moleculardynamics (MD) simulations based on a classical potential model fit to first-principles calculations for ordered $\mathrm{Fe}-\mathrm{Cu}$ compounds. The theoretical results in Fig. 7 are plotted as 
TABLE II. A comparison between current and previous first-principles calculations and experimental measurements for atomic volumes, magnetic moment, and elastic constants of pure $\mathrm{Cu}$ and Fe. FLAPW stands for the full-potential linearized augmented plane-wave method. PAW represents a projector augmented wave method. USPP (VASP) stands for ultrasoft pseudopotentials, as implemented in VASP. The calculations are performed with a GGA or LDA exchange-correlation potential, which includes PW91 (Ref. 54), PBE96 (Ref. 59), PW92 (Ref. 60), CA (Ref. 61 and 62) and Wigner (Ref. 63). Both scalar-relativistic (SR) and nonrelativistic (NR) calculated results from Ref. 64 are included.

\begin{tabular}{|c|c|c|c|c|c|c|c|c|}
\hline & Method & $\begin{array}{l}\text { Exchange } \\
\text { correlation }\end{array}$ & $\begin{array}{c}\text { Atomic volume } \\
\qquad\left(\AA^{3}\right)\end{array}$ & $\begin{array}{l}\text { Magnetic moment } \\
\quad\left(\mu_{B} / \text { atom }\right)\end{array}$ & $B(\mathrm{GPa})$ & $C_{44}(\mathrm{GPa})$ & $C^{\prime}(\mathrm{GPa})$ & Reference \\
\hline \multirow[t]{13}{*}{$\mathrm{Fe} \mathrm{bcc}$} & PAW (VASP) & GGA PW91 & 11.3 & 2.16 & 194 & 101 & 69 & This work \\
\hline & USPP (VASP) & GGA PW91 & 11.5 & 2.32 & 176 & 115 & 85.5 & 65 \\
\hline & USPP (VASP) & GGA PW91 & 11.7 & 2.32 & 160 & & & 31 \\
\hline & LMTO-ASA & GGA PW91 & 11.4 & 2.24 & 176 & & & 66 \\
\hline & FLAPW & GGA PW91 & 11.6 & 2.32 & 169 & & & 67 \\
\hline & FLAPW & GGA PW91 & 11.4 & 2.17 & 189 & & & 68 \\
\hline & FLAPW & GGA PW91 & 11.5 & & 180 & & & 69 \\
\hline & FLAPW & GGA PW91 & 11.7 & & 171 & & & 32 \\
\hline & FLAPW & GGA PW91 & 11.8 & 2.29 & & & & 30 \\
\hline & FLAPW & GGA PW91 & 11.5 & 2.20 & & & & 29 \\
\hline & FLAPW & GGA PBE96 & 11.4 & 2.17 & 186 & 99 & 69.5 & Ref. 70 \\
\hline & FLAPW & GGA PBE96 & 11.6 & 2.14 & 188 & 99.5 & 73 & 71 \\
\hline & Experiment $(\sim 4 \mathrm{~K})$ & & 11.7 & $2.20,2.23$ & 173 & 122 & 52.5 & $\mathrm{R} 15,17,57$, and 58 \\
\hline \multirow[t]{9}{*}{$\mathrm{Cu} \mathrm{bcc}$} & PAW (VASP) & GGA PW91 & 12.1 & & 133 & 95.9 & -14.5 & this work \\
\hline & PAW (VASP) & GGA PW91 & 12.0 & & & & & 72 \\
\hline & USPP (VASP) & GGA PW91 & 12.1 & & 160 & & & 31 \\
\hline & FLAPW & LDA PW92 & 11.0 & & 188 & 112 & -6 & 36 and 37 \\
\hline & FLAPW & GGA PW91 & 12.2 & & 141 & & & 32 \\
\hline & FLAPW & GGA PW91 & 12.2 & & & & & 30 \\
\hline & FPLMTO & LDA CA & 11.0 & & & 69 & & 34 \\
\hline & FLAPW (SR) & LDA Wigner & 11.5 & & 179 & & & 64 \\
\hline & FLAPW (NR) & LDA Wigner & 11.7 & & 160 & & & 64 \\
\hline \multirow[t]{6}{*}{$\mathrm{Cu} \mathrm{fcc}$} & PAW (VASP) & GGA PW91 & 12.0 & & 134 & 64.5 & 29.9 & This work \\
\hline & USPP (VASP) & GGA PW91 & 12.1 & & 140 & & & 31 \\
\hline & FLAPW & GGA PW91 & 11.9 & & 179 & & & 32 \\
\hline & FLAPW & GGA PW91 & 12.2 & & & & & 30 \\
\hline & FLAPW & GGA PW91 & 11.8 & & & & & 29 \\
\hline & Experiment $(0 \mathrm{~K})$ & & 11.7 & & 142 & 81.8 & 25.6 & 58 and 73 \\
\hline
\end{tabular}

formation energies, referenced to bcc Fe and fcc $\mathrm{Cu}$, to compare with the measured values of the enthalpies of formation. The agreement between the present calculations and experimental measurements is seen to be very good, with a maximum discrepancy of about $0.02 \mathrm{eV} /$ atom for the most concentrated alloy. A similar level of agreement is found with the calculations by Gong et al., ${ }^{33}$ which are seen to be slightly larger in magnitude for near-equiatomic compositions.

The atomic volumes $\left(V_{0}\right)$ for $\mathrm{Fe}-\mathrm{Cu}$ solid solutions plotted in Fig. 2 show a pronounced positive deviation from linearmixing behavior, consistent with experimental measurements for metastable $\mathrm{Fe}-\mathrm{Cu}$ alloys. ${ }^{15-18,20,22,23}$ The origin of this behavior has been discussed in the theoretical work by Zhang and $\mathrm{Ma},{ }^{30}$ who show that the positive deviation from linear-mixing behavior is associated with the coupling between magnetism and atomic volume. A comparison between experimental measurements and the present calculations is given in Fig. 8, which plots the formation volume $\Delta V_{0}$ versus concentration for bcc $\mathrm{Fe}-\mathrm{Cu}$ solid solutions. The quantity $\Delta V_{0}$ for an $\mathrm{Fe}_{1-x} \mathrm{Cu}_{x}$ alloy is defined analogously to the formation energy as the difference between the alloy volume and the concentration-weighted average of the volumes for elemental bcc $\mathrm{Fe}$ and fcc $\mathrm{Cu}$. Figure 8 compares this quantity with measurements, rather than $V_{0}$ itself, to remove discrepancies associated with the underestimation of the atomic volume for bcc Fe by the GGA, and to focus on the predictions for concentration dependencies. Although the experimental data shows considerable scatter, the theoretical predictions for $\Delta V_{0}$ are of comparable magnitude to the measurements (note that the early data of Kneller, ${ }^{15}$ which also showed positive deviations from Vegard's law, was not included in Fig. 8 since it showed considerable scatter). It is noteworthy that we find particularly good quantitative agreement with 
TABLE III. Atomic volume, bulk modulus and formaton energy for three selected bcc-based $\mathrm{Fe}-\mathrm{Cu}$ compounds, comparing the results of the present and previous first-principles calculations.

\begin{tabular}{|c|c|c|c|c|c|c|}
\hline & Method & Exchange-Correlations & $\begin{array}{l}\text { Atomic Volume } \\
\qquad\left(\AA^{3}\right)\end{array}$ & $\mathrm{B}(\mathrm{GPa})$ & $\Delta E_{\text {form }}(\mathrm{eV} /$ atom $)$ & Reference \\
\hline \multirow[t]{4}{*}{$\mathrm{B} 2 \mathrm{FeCu}$} & FLAPW & GGA PW91 & 12.2 & & & 30 \\
\hline & FLAPW & GGA PW91 & 12.2 & & & 29 \\
\hline & PAW (VASP) & GGA PW91 & 12.1 & 139 & 0.275 & 33 \\
\hline & PAW (VASP) & GGA PW91 & 12.0 & 134 & 0.281 & This work \\
\hline \multirow[t]{3}{*}{$\mathrm{DO}_{3} \mathrm{FeCu}_{3}$} & FLAPW & GGA PW91 & 12.4 & & & 30 \\
\hline & PAW (VASP) & GGA PW91 & 12.1 & & & 33 \\
\hline & PAW (VASP) & GGA PW91 & 12.1 & 143 & 0.180 & This work \\
\hline \multirow[t]{4}{*}{$\mathrm{DO}_{3} \mathrm{Fe}_{3} \mathrm{Cu}$} & FLAPW & GGA PW91 & 11.98 & & & 30 \\
\hline & FLAPW & GGA PW91 & 12.0 & & & 29 \\
\hline & PAW (VASP) & GGA PW91 & 12.0 & & 0.153 & 33 \\
\hline & PAW (VASP) & GGA PW91 & 11.8 & 166 & 0.162 & This work \\
\hline
\end{tabular}

the most recent measurements due to Ma et $a l .^{23}$

In Fig. 3 the present $a b$ initio results for magnetic moment of ordered bcc-based $\mathrm{Fe}-\mathrm{Cu}$ compounds are plotted with open symbols; the filled symbols represent the clusterexpansion fit, while the solid line is the cluster-expansion prediction for random bcc solid solutions. The ab initio results are seen to display a highly linear dependence on $\mathrm{Cu}$ concentration, and are well fit by a cluster expansion containing only the point and one pair cluster. These results are in good agreement with previous first-principles investigations ${ }^{29,30}$ and confirm the noted trend that the magnetic moment is highly insensitive to the atomic configuration at fixed composition. In Fig. 9 the cluster-expansion predictions are compared with experimental measurements of the magnetic moment for bcc Fe-Cu solid solutions. 15,17,23 Overall, the agreement is seen to be quite good, with the cluster-expansion results being slightly larger at the higher $\mathrm{Cu}$ concentrations. The temperature-dependent data of Kneller ${ }^{15}$ and Sumiyama et al. ${ }^{17}$ shows a clear and expected trend toward increasing magnetic moment with decreasing temperature, and it is noteworthy that our zero-temperature results show the best agreement with the $4.2 \mathrm{~K}$ data.

TABLE IV. Details related to the cluster-expansion fits for the mixing energy $\left(\Delta E_{m i x}\right)$, atomic volume $\left(V_{0}\right)$, magnetic moment $(M)$, bulk modulus $(B)$, and the symmetrized moduli $\overline{C^{\prime}}$ and $\bar{C}_{44} . N$ is the number of structures used in the SIM fitting. Here $\mu_{B}$ stands for Bohrs magneton.

\begin{tabular}{lccccc}
\hline \hline & & \multicolumn{3}{c}{ Number of clusters } & \\
\cline { 3 - 5 } Property & $N$ & Pair & Triplet & Quadruplet & CV score \\
\hline$\Delta E_{\text {mix }}$ & 28 & 7 & 2 & & $0.013 \mathrm{eV} /$ atom \\
$V_{0}$ & 28 & 4 & 6 & 2 & $0.10 \AA^{3}$ \\
$B$ & 28 & 4 & 6 & 2 & $4.01 \mathrm{GPa}$ \\
$\bar{C}^{\prime}$ & 18 & 4 & 5 & 2 & $4.81 \mathrm{GPa}$ \\
$\bar{C}_{44}$ & 17 & 4 & & & $6.71 \mathrm{GPa}$ \\
$M$ & 30 & 1 & & & $0.0850 \mu_{B} /$ atom \\
\hline \hline
\end{tabular}

The cluster expansion predictions for the elastic moduli of disordered bcc $\mathrm{Fe}-\mathrm{Cu}$ solid solutions are plotted with solid lines in Figs. 4, 5, and 6. The cluster-expansion results show significant negative deviations from linear mixing behavior for the bulk modulus and $C^{\prime}$, while $C_{44}$ is predicted to display a weak positive deviation from linearity. Of particular interest in the current work is the predicted concentration dependence of $C^{\prime}$, which is computed to be positive in the $\mathrm{Fe}$-rich region and negative for $\mathrm{Cu}$-rich alloys. The $\mathrm{Cu}$-rich bcc solid solution is thus predicted to be mechanically unstable over a wide concentration range. Outside of that range, the mechanical stability (i.e., the magnitude of $C^{\prime}$ ) of the alloy increases sharply with increasing $\mathrm{Fe}$ concentration greater than 50 atomic \%. This behavior is very similar to that obtained for $C^{\prime}$ in theoretical calculations for the $\mathrm{Ni}-\mathrm{Cr}$

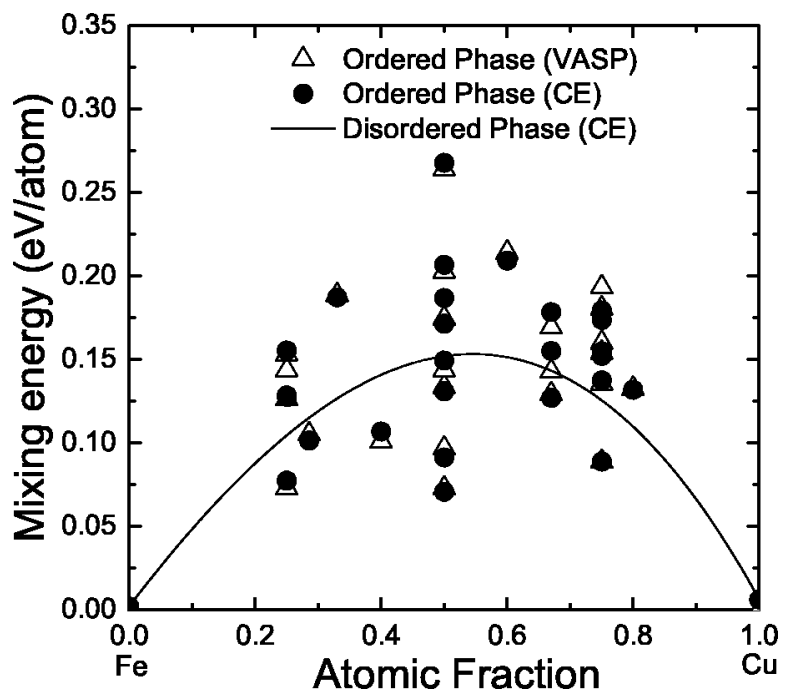

FIG. 1. Mixing energy versus $\mathrm{Cu}$ concentration in $\mathrm{Fe}-\mathrm{Cu}$ bcc alloys. The symbols represent the values for selected ordered structures. Open triangles are the $a b$ initio calculations (VASP) while the solid circles are the predictions from the cluster expansion (CE). The solid curve is the $\mathrm{CE}$ prediction for a random disordered bcc solid-solution phase. 


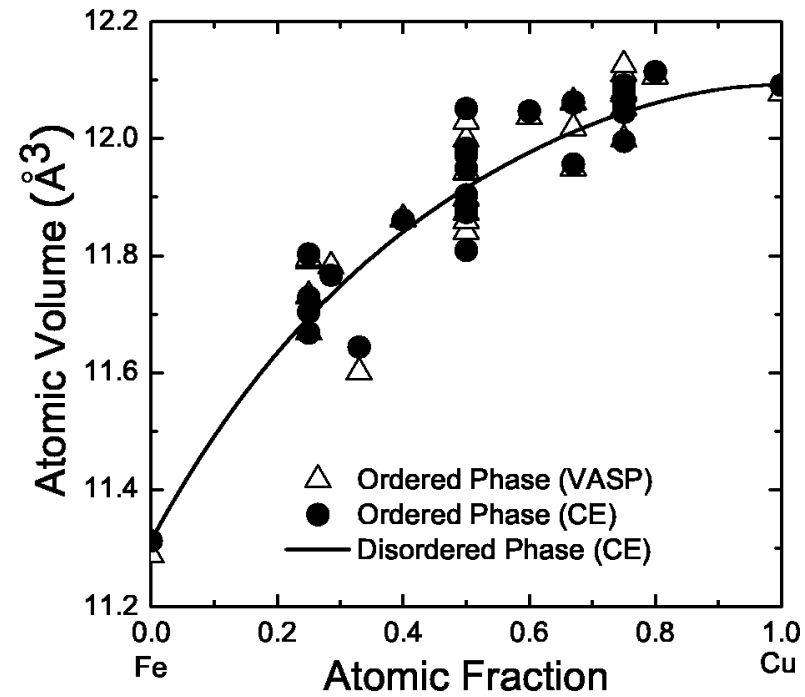

FIG. 2. Atomic volume versus $\mathrm{Cu}$ concentration in bcc $\mathrm{Fe}-\mathrm{Cu}$ alloys. The symbols represent the values for selected ordered structures. Open triangles are the $a b$ initio results (VASP) while the solid circles are the values predicted by cluster expansion (CE). The solid line is the $\mathrm{CE}$ prediction for a random disordered bcc $\mathrm{Fe}-\mathrm{Cu}$ solidsolution phase.

system by Craievich et al. ${ }^{40,41}$ where the bcc solid solution is stable only for relatively high $\mathrm{Cr}$ concentrations.

\section{DISCUSSION}

\section{A. Stability of bec Fe-Cu solid solutions}

A central result of the present study concerns the relatively extended width of the unstable region (i.e., the region of concentration with negative $C^{\prime}$ ) for bcc $\mathrm{Fe}-\mathrm{Cu}$ solid solu-

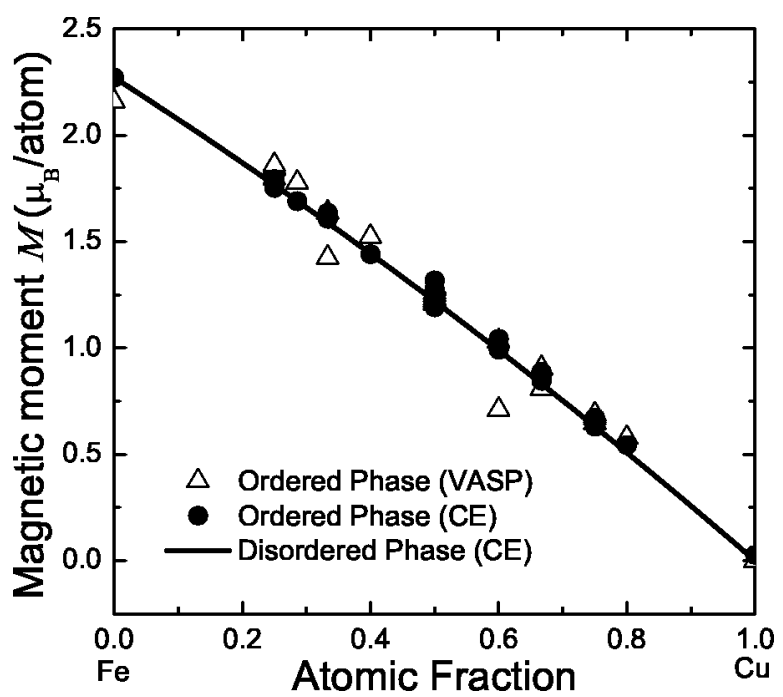

FIG. 3. Magnetic moment per atom $(M)$ versus $\mathrm{Cu}$ concentration for bcc-based $\mathrm{Fe}-\mathrm{Cu}$ alloys. Open triangles represent the results from $a b$ initio (VASP) calculations for selected ordered structures. Solid circles represent the predictions from the cluster expansion (CE). The solid line is the CE prediction for random disordered bcc $\mathrm{Fe}-\mathrm{Cu}$ solid solutions.

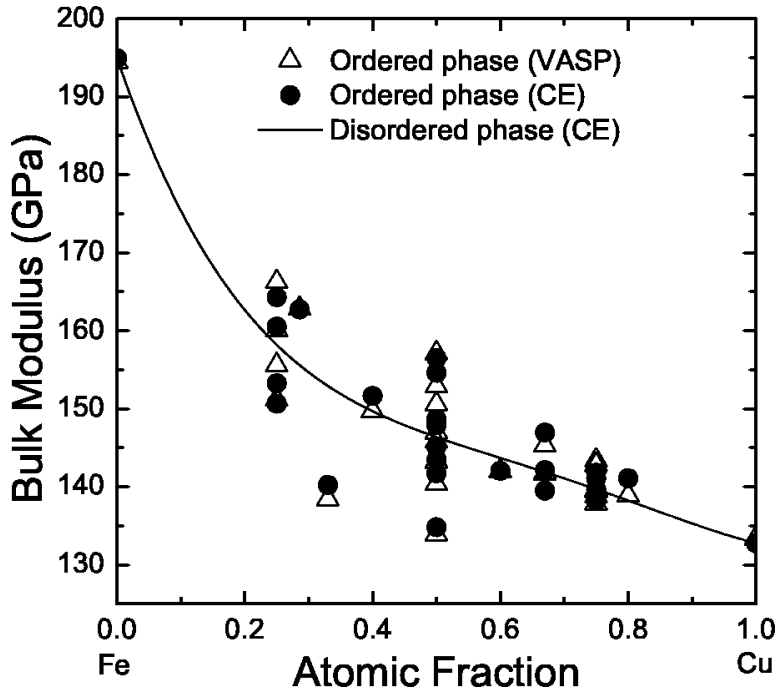

FIG. 4. Bulk modulus versus $\mathrm{Cu}$ concentration in bcc $\mathrm{Fe}-\mathrm{Cu}$ alloys. The symbols represent the values for selected ordered structures. Open triangles are the $a b$ initio results (VASP) while the solid circles are the values predicted by cluster expansion (CE). The solid line is the $\mathrm{CE}$ prediction for the random disordered bcc $\mathrm{Fe}-\mathrm{Cu}$ solidsolution phase.

tions plotted in Fig. 5. This result, and the highly nonlinear concentration dependence, are entirely compatible with existing semiempirical $d$-band models describing the relative stability of different lattice types across the transition metal series (see Ref. 75 and references therein). The basic picture is analogous to the Jahn-Teller mechanism, where, above a critical number of electrons, a degenerate energy level becomes partially occupied. The energy of the system can therefore be reduced by splitting this level, which can be

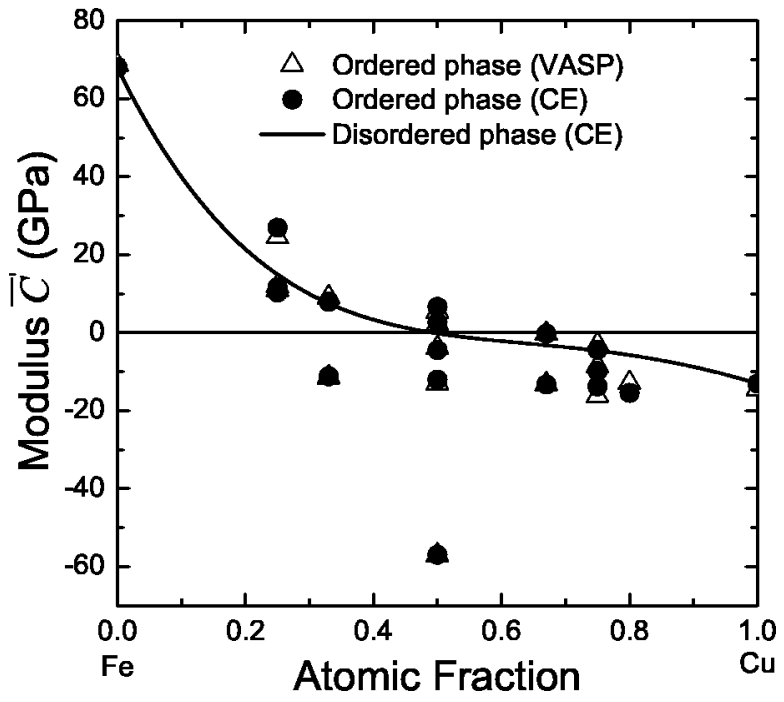

FIG. 5. Tetragonal shear modulus $\bar{C}^{\prime}$ (symmetrized) versus $\mathrm{Cu}$ concentration in bcc $\mathrm{Fe}-\mathrm{Cu}$ alloys. The symbols represent the values for selected ordered structures. Open triangles are the $a b$ initio results (VASP) while the solid circles are the values predicted by cluster expansion (CE). The solid line is the $\mathrm{CE}$ prediction for a random disordered bcc $\mathrm{Fe}-\mathrm{Cu}$ solid-solution phase. 


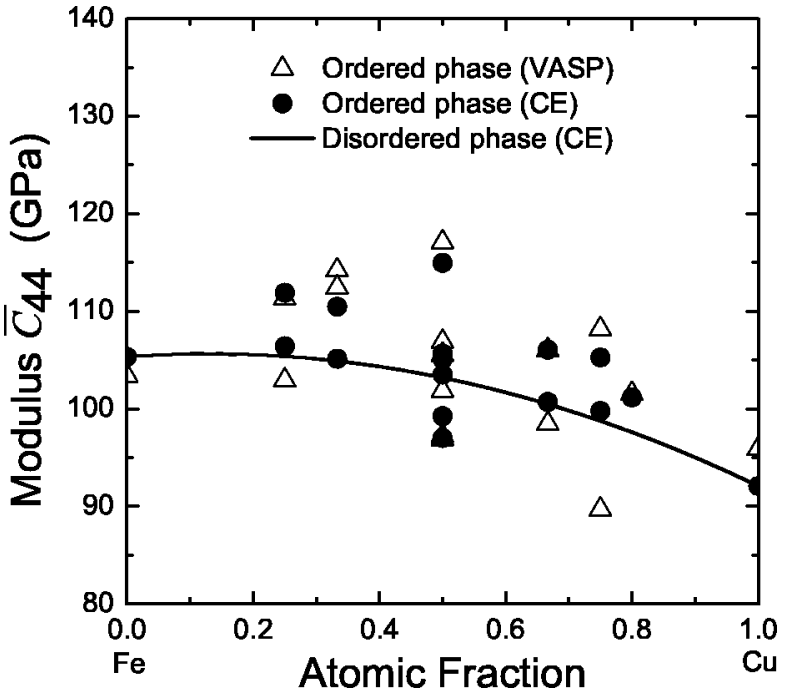

FIG. 6. Modulus $\bar{C}_{44}$ (symmetrized) versus $\mathrm{Cu}$ concentration in bcc $\mathrm{Fe}-\mathrm{Cu}$ alloys. The symbols represent the values for selected ordered structures. Open triangles are the $a b$ initio results (VASP) while the solid circles are the values predicted by the cluster expansion $(\mathrm{CE})$. The solid line is the $\mathrm{CE}$ prediction for a random disordered bcc Fe-Cu.

achieved by breaking the bcc symmetry. This mechanism helps explain why the transition to mechanical stability is so nonlinear. As seen in Fig. 12 in Ref. 75, the critical electron number in elemental solids is about $6 d$ electrons per atom, placing the transition at the electron concentration corresponding to pure Fe. This picture thus clarifies why bcc remains unstable until a large amount of $\mathrm{Fe}$ is added to the solution. Of course, this picture is mostly qualitative, be-

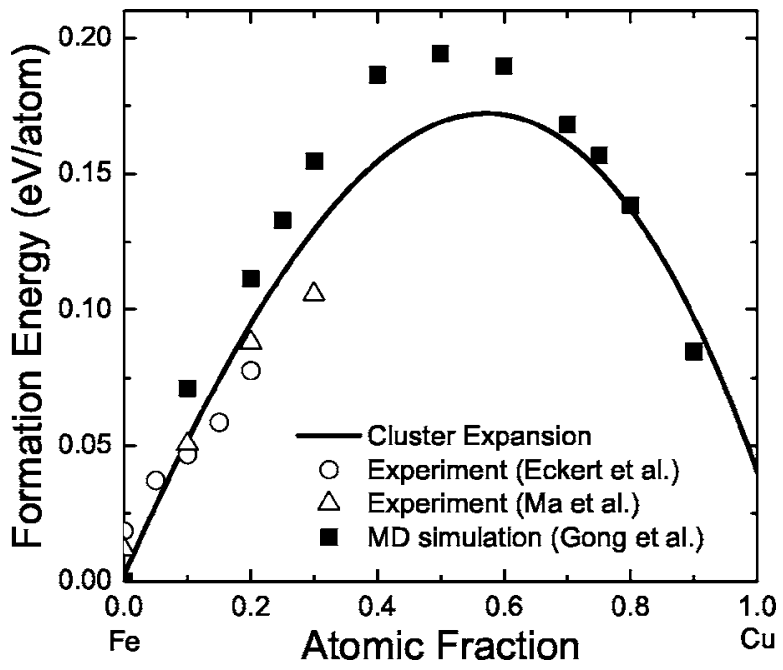

FIG. 7. Formation energy versus $\mathrm{Cu}$ concentration in $\mathrm{Fe}-\mathrm{Cu}$ bcc solid solutions. Open circles and triangles represent experimental measurements performed on metastable alloys obtained through mechanical alloying from Eckert et al. (Ref. 22) and Ma et al. (Ref. 23), respectively. Solid squares correspond to theoretical results derived from molecular dynamics simulations by Gong et al. (Ref. 33). The solid curve represents the current theoretical prediction based on the cluster expansion (CE) approach.

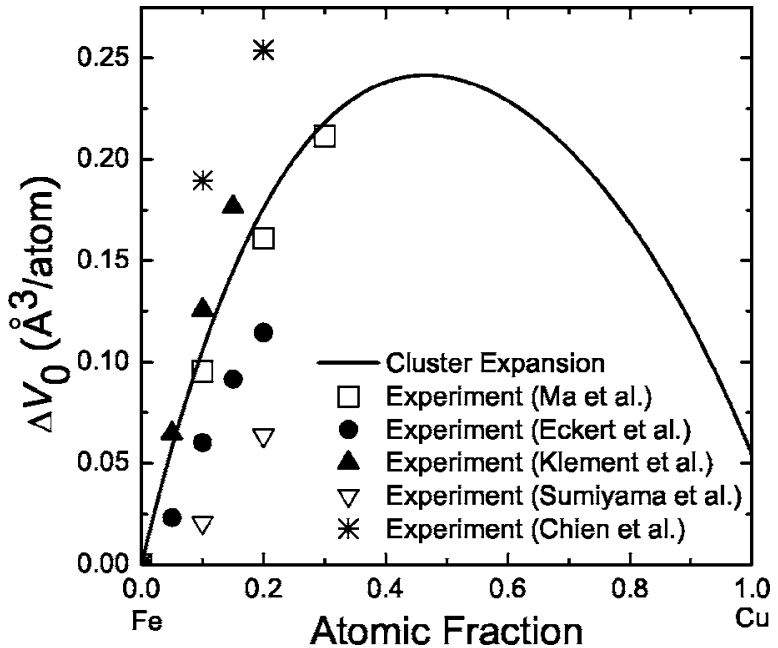

FIG. 8. Formation volume $\Delta V_{0}$ versus $\mathrm{Cu}$ concentration for bcc $\mathrm{Fe}-\mathrm{Cu}$ solid solutions. Open squares and solid circles are experimental results from mechanically-alloyed samples due to Ma et al. (Ref. 23) and Eckert et al. (Ref. 22), respectively. Solid triangles are experimental measurements from alloys obtained by rapid quenching by Klement et al. (Ref. 16). Open triangles and crosses are experimental results from sputter-deposited samples due to Sumiyama et al. (Ref. 17) and Chien et al. (Ref. 18). The current theoretical predictions, obtained from the cluster-expansion approach, are given by the solid lines.

cause both the magnetic nature of $\mathrm{Fe}$ and the focus on a random alloy, rather than on a pure element, substantially complicate the analysis. In fact, these complications further emphasize the need for the accurate $a b$ initio treatment presented above.

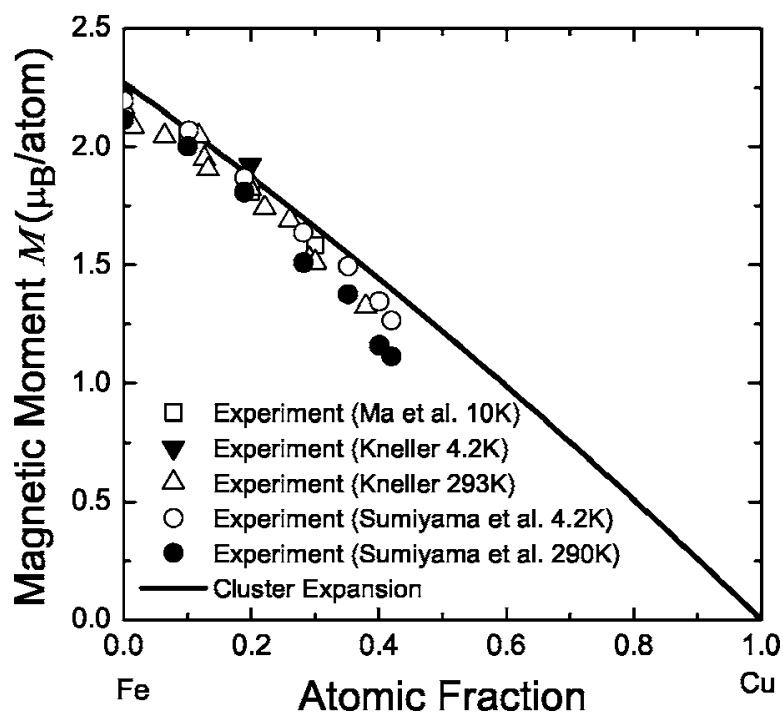

FIG. 9. Measured and calculated magnetic moments (per atom) in bcc $\mathrm{Fe}-\mathrm{Cu}$ solid solutions. Open squares are measured results from mechanically alloyed samples due to Ma et al. (Ref. 23). Solid and open triangles represent measurements from vapor-deposited samples at temperatures of 4.2 and $293 \mathrm{~K}$, respectively, due to Kneller (Ref. 15). Solid and open circles are measurements from sputter-deposited alloys due to Sumiyama et al. (Ref. 17). The solid line represents the present calculated results for random $\mathrm{Fe}-\mathrm{Cu}$ bcc solid solutions. 
TABLE V. Summary of experimental measurements for the diameter and $\mathrm{Fe}$ concentraton of precipitates in a binary $\mathrm{Fe}-\mathrm{Cu}$ alloy.

\begin{tabular}{lccc}
\hline \hline Method & Diameter (nm) & Fe concentration (at \%) & Reference \\
\hline AP $^{\mathrm{a}}$ & 1.7 & 51 & 12 \\
& 2.7 & 54 & \\
& 3.0 & 45 & 6 \\
& 5.0 & 32 & 76 \\
PAS $^{\mathrm{b}}$ & & $<10$ & 77 \\
SANS $^{c}$ & 4.1 & 0 & \\
& 4.4 & 0 & \\
\hline
\end{tabular}

${ }^{a}$ Conventional (one-dimensional) atom probe.

${ }^{\mathrm{b}}$ Positron annihilation spectroscopy.

${ }^{\mathrm{c}}$ Small angle neutron scattering.

It is interesting to analyze experimental observations concerning the stability of bcc $\mathrm{Fe}-\mathrm{Cu}$ alloys in light of the present results. Through the nonequilibrium processing technique of mechanical alloying, it has been possible to synthesize $\mathrm{Fe}-\mathrm{Cu}$ solid-solution phases over the entire concentration range. As reviewed by Zhang and $\mathrm{Ma},{ }^{30}$ it is generally observed in such studies that bcc solid solutions are only observed for $\mathrm{Cu}$ concentrations below 40 atomic \%; at higher $\mathrm{Cu}$ concentrations only the fcc structure is observed. These observations have been analyzed in previous studies employing models for the free-energy differences between the competing fcc and bcc solid solutions, ${ }^{23,33}$ and the observed ranges of stability have been correlated well with the crossing of the free energy curves for these competing phases with respect to concentration. In light of the current work, it is important to emphasize that these previous analyses have been based either on calculations of energy alone, i.e., neglecting entropic contributions, or on free-energy models employing an ideal entropy of mixing. The results in Fig. 5, featuring negative values for $C^{\prime}$ for $\mathrm{Cu}$-rich compositions, suggest that the thermodynamics of bcc alloys, and thus the phase competition between fcc and bcc solid solutions, is substantially more complex. Specifically, due to the small and negative values of $C^{\prime}$ for $\mathrm{Cu}$-rich alloys, realistic models of the thermodynamics of bcc $\mathrm{Fe}-\mathrm{Cu}$ alloys should account for anharmonic vibrational-entropy effects and the possibility that the bcc structure is inherently unstable beyond a critical $\mathrm{Cu}$ concentration.

In supersaturated $\mathrm{Fe}-\mathrm{Cu}$ alloys, nanometer-scale precipitates are known to form with the bcc crystal structure for diameters less than 3-4 nm. ${ }^{6-8,11}$ The concentration of these precipitates has been a topic of debate, with alternate experimental methods leading to significantly different reported compositions, as summarized in Table V. In this table it is seen that direct measurements based on the technique of atom-probe microscopy consistently yield precipitate concentrations with high $\mathrm{Fe}$ concentrations, in the range of 50 atomic $\%$ for sizes below $4 \mathrm{~nm} .^{6,12}$ By contrast indirect derived values based on both positron-annihilation and neutron-scattering techniques give much lower $\mathrm{Fe}$ concentrations. $^{76,77}$ It is interesting to note that the atomprobe measurements for bcc precipitates correspond well with the limiting Fe concentration for the mechanical stabil- ity of the bcc solid-solution phase predicted in the present study. In other words, the high concentrations of Fe reported by atom-probe microscopy could be understood, in light of the results in Fig. 5, as having the effect of stabilizing the bcc structure of the precipitate. If the precipitates in fact have the higher $\mathrm{Cu}$ concentrations suggested by neutron scattering and positron annihilation, other factors would need to be considered to reconcile the results in Fig. 5 with the observed stability of the bcc structure in small coherent precipitates. One such possibility is that, in a $\mathrm{Cu}$-rich precipitate, the bcc structure could be stabilized by the strains imposed by the constraint of coherency with the higher-density Fe-rich matrix. To investigate this possibility, we performed $a b$ initio calculations of $C^{\prime}$ for bcc $\mathrm{Cu}$ at the atomic volume of bcc Fe, finding an increase to $-12.0 \mathrm{GPa}$ from the equilibrium value of $-14.5 \mathrm{GPa}$. While this strain effect has the right sign, it does not appear to be of sufficient magnitude to stabilize a $\mathrm{Cu}$-rich bcc structure. An additional factor that could promote the stability of a bcc structure in a small coherent precipitate are the epitaxial forces imposed by the interfacial bonds with the bcc matrix. For example, to explain the observation of the mechanically unstable bcc structure in epitaxially grown $\mathrm{Cu}$ films on $\mathrm{Fe}$ and $\mathrm{Ag}$ (001) substrates, ${ }^{38,39}$ Kraft et al. ${ }^{34}$ have suggested that due to the epitaxial constraint on $\{001\}$ surfaces the spontaneous sliding on $\{011\}$ planes will be prevented since $C_{44}$ is positive for bcc $\mathrm{Cu}$. Additionally, it is possible that anharmonic vibrational effects could lead to a stabilization of $C^{\prime}$ at finite temperature. Further experimental and theoretical work is clearly warranted to resolve the controversy surrounding precipitate concentrations and the associated origins for the stability of the bcc structure in these nanoscale particles.

\section{B. Implications for the strengthening mechanism}

The pronounced strengthening effect of nanoscale bcc precipitates in $\mathrm{Fe}-\mathrm{Cu}$ alloys has traditionally been attributed to a modulus effect, as first proposed in the work of Russel and Brown. ${ }^{78}$ In this model, precipitates with a softer shear modulus than the matrix provide an attractive interaction with dislocations and thus act to "pin" their motion. A key parameter in the Russel-Brown model is the ratio of the isotropic shear modulus in the precipitate $\left(\mu_{1}\right)$ and matrix $\left(\mu_{2}\right)$. In applying the model to $\mathrm{Fe}-\mathrm{Cu}$ alloys Russel and Brown estimated $\mu_{1}$ as the measured isotropic shear modulus for fcc $\mathrm{Cu}$, since values for bcc $\mathrm{Cu}$ were not available. This yields a ratio $\mu_{1} / \mu_{2}=0.6$, with which Russel and Brown demonstrated very reasonable agreement between their theory and available experimental measurements for the dependence of peak hardness on precipitate volume fraction, provided the one remaining free parameter, namely the dislocation core radius $\left(r_{0}\right)$, was fit with a value of roughly twice the Burger's vector $(b)$. With these values for $\mu_{1} / \mu_{2}$ and $r_{0}$, the RusselBrown theory predicts that the precipitate radius at peak strength is approximately twice $r_{0}$, or about $1 \mathrm{~nm}$. It was noted by Russel and Brown that this result is about a factor of 2 smaller than the measured values for average precipitate radii at peak hardness.

With the present results for the single-crystal elastic constants of bcc $\mathrm{Fe}-\mathrm{Cu}$ alloys, it is interesting to revisit the 


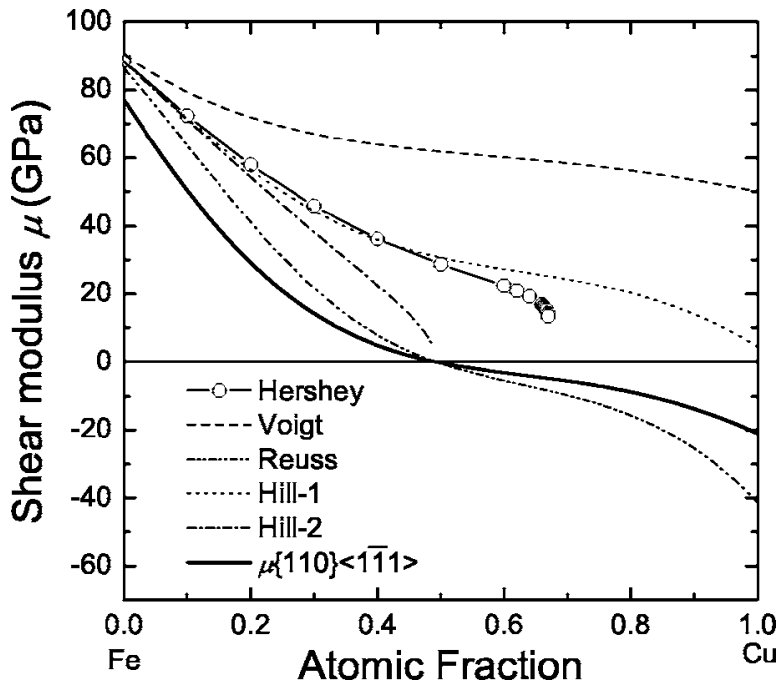

FIG. 10. Isotropic shear modulus $\mu$ and shear modulus $\mu_{\{110\}\langle 1 \overline{1} 1\rangle}$ on primary slip plane $\{110\}$ versus $\mathrm{Cu}$ concentration for random bcc $\mathrm{Fe}-\mathrm{Cu}$ solid solutions predicted from the present calculations.

analysis of Russel and Brown for $\mathrm{Fe}-\mathrm{Cu}$ alloys. A more complete analysis will be given in a separate publication; here the results are only briefly summarized. In applying the model of Russel and Brown to $\mathrm{Fe}-\mathrm{Cu}$ alloys, the appropriate shear modulus is that for the primary slip plane $\{110\}::^{79}$

$$
\mu_{\{110\}\langle 1 \overline{1} 1\rangle}=\frac{3 C_{44}\left(C_{11}-C_{12}\right)}{C_{11}-C_{12}+4 C_{44}}
$$

Figure 10 plots the concentration dependence of this quantity, as derived from the results in Figs. 4-6. We also include in Fig. 10 plots of the isotropic shear modulus computed according to the prescriptions of Voigt, Reuss, and Hershy, according to the equations listed in the Appendix; these moduli find wide application in the literature on precipitation and are thus included for completeness. If we assume that the precipitates have a concentration near 50 at. $\% \mathrm{Fe}$, as reported by atom-probe microscopy, ${ }^{6,12}$ the results in Fig. 10 predict that the ratio $\mu_{1} / \mu_{2}$ is approximately zero. If this value is employed in the Russel-Brown model, we obtain the same agreement with the measured volume-fraction dependence of shear strength, as previously reported ${ }^{78}$ provided we assume a significantly larger value for the core radius of $r_{0} \sim 4 b$. This result leads to a predicted precipitate size at peak strength of approximately $2 \mathrm{~nm}$, in good agreement with measurements.

Recently, the ability of the Russel-Brown model to explain the precipitate-strengthening mechanism in $\mathrm{Fe}-\mathrm{Cu}$ alloys has been called into question..$^{2-4,80}$ In Ref. 4, Fine and Isheim obtained an inaccurate estimate of the level of strengthening arising from the Russel-Brown formulation of modulus-mismatch hardening from precipitates with vanishingly small shear moduli, due to the use of an incorrect formula; this has been corrected in the present analysis that yields a substantial contribution to hardening from modulus mismatch.
It has also been noted by Harry and Bacon ${ }^{2}$ that (i) empirical-potential models ${ }^{81,82}$ predict that the shear modulus for bulk bcc $\mathrm{Cu}$ is actually larger than that of $\alpha$-Fe. These potentials also predict that while bulk bcc $\mathrm{Cu}$ is stable at zero presssure, (ii) it becomes unstable with respect to shear under hydrostatic compression. Harry and Bacon ${ }^{2}$ have concluded based on these predictions that there is significant doubt that the component of hardening due to modulus mismatch is significant compared with the measured increases in yield strength. These authors propose a novel alternative strengthening mechanism, based on a detailed analysis of atomistic simulations employing the potentials of Ref. 82, which is related to the transformation of the unstable bcc $\mathrm{Cu}$ lattice to a close-packed structure when it is sheared by a dislocation. It is important to point out that both predictions (i) and (ii), which arise from classical-potential models, are inconsistent with the present first-principles results. As detailed above, the present calculations suggest that the hardening due to modulus mismatch is likely to be significant. It should be noted, however, that in the analysis leading to this conclusion we have used the precipitate compositions reported from atom-probe measurements, which have high enough Fe concentrations to lead to a positive value of $\mu_{1}$. If the precipitates have a much lower Fe concentration, as suggested by PAS and SANS measurements, and thus negative $\mu_{1}$, the structural-transformation mechanism proposed by Harry and Bacon may indeed be relevant. It would thus be of interest to refit empirical potential models for $\mathrm{Fe}-\mathrm{Cu}$ employing the present and previous first-principles results as a basis for further atomistic-simulation work of the type reported in Ref. 2 to examine the nature of the hardening mechanisms in more detail. In such an analysis, the present results (e.g., Fig. 10) point to the importance of considering deviations in the precipitate concentration away from pure $\mathrm{Cu}$.

\section{SUMMARY AND CONCLUSIONS}

$A b$ initio calculations, in conjunction with the cluster expansion framework, were used to compute the composition dependencies of the atomic volume, mixing energy, magnetic moment, and elastic moduli of disordered bec solid solutions in the $\mathrm{Fe}-\mathrm{Cu}$ system. While cluster expansions are traditionally used to represent the configuration and concentration dependence of scalar quantities, we have demonstrated how the same formalism can be extended to the calculation of the elastic constants for disordered solid solutions. The mixing energy and atomic volume obtained in the present calculations both deviate positively from linear-mixing behavior, and are in good quantitative agreement with available experimental measurements. The calculated magnetic moment displays a highly linear dependence on the $\mathrm{Cu}$ concentration and agrees well with experimental measurements. The bulk modulus $B$ and shear modulus $C_{44}$ are both positive throughout the entire concentration range, while $C^{\prime}$ is calculated to be negative in the $\mathrm{Cu}$-rich region, indicating that bcc solid solutions with $\mathrm{Cu}$-rich concentrations are mechanically unstable. The present results suggest that the thermodynamic properties of bcc $\mathrm{Fe}-\mathrm{Cu}$ solid solutions are likely to contain a sizable contribution from vibrational entropy, which itself is 
TABLE VI. Isotropic shear modulus of a bcc Fe polycrystal. Each quantity is expressed in the units of $\mathrm{GPa}$.

\begin{tabular}{lcccccc}
\hline \hline & Temperature (K) & $\mu_{V}$ & $\mu_{R}$ & $\mu_{H i l l-1}$ & $\mu_{H i l l-2}$ & $\mu_{H}$ \\
\hline This work & 0 & 88.4 & 85.3 & 86.9 & 86.8 & 87.0 \\
Experiment (Ref. 57) (single crystal) & 4.2 & 94.1 & 79.7 & 86.9 & 86.6 & 87.5 \\
Experiment (Ref. 88) (polycrystal) & 77 & & 85.5 & & & \\
\hline \hline
\end{tabular}

expected to be highly anharmonic in nature. Due to the low values of $C^{\prime}$ for bcc alloys with the precipitate compositions measured by atom-probe microscopy, we conclude that the Russel-Brown modulus effect is likely to represent a sizeable contribution to the measured strengthening effect of nanoscale bcc precipitates in $\mathrm{Fe}-\mathrm{Cu}$.

\section{ACKNOWLEDGMENTS}

This research was supported by the Office of Naval Research Grant No. N00014-03-1-0252/P00002, Dr. Julie A. Christodoulou Program Director. The authors thank Professor M. E. Fine and Dr. D. Isheim at Northwestern for numerous helpful discussions and input.

\section{APPENDIX: ISOTROPIC SHEAR MODULUS}

For anisotropic cubic structures there are several methods to estimate the isotropic shear modulus $(\mu)$ for a totally random polycrystal from the single crystal elastic constants $C_{i j}$, including the methods of Voigt, ${ }^{83}$ Reuss, ${ }^{84}$ Hill, ${ }^{85}$ and Hershey. ${ }^{86}$ In Voigt's ${ }^{83}$ method $\left(\mu_{V}\right)$, based on the assumption of uniform local strain, the isotropic shear modulus is expressed as

$$
\mu_{V}=\frac{C_{11}-C_{12}+3 C_{44}}{5}
$$

On the other hand, in Reuss's ${ }^{84}$ method $\left(\mu_{R}\right)$, based on the assumption of uniform local stress, the isotropic shear modulus is expressed as

$$
\mu_{R}=\frac{5}{4\left(S_{11}-S_{12}\right)+3 S_{44}},
$$

where $S_{i j}$ 's are the elastic compliances. Despite their wide usage, neither Voigt's nor Reuss' relation is believed to be exact. Accordingly, Hill ${ }^{85}$ showed that Voigt's equation provides an upper limit for $\mu$ while Reuss's equation gives a lower limit for $\mu$. Hill ${ }^{85}$ suggested averaging by either the arithmetic or the geometric mean of these two limits, i.e.,

$$
\mu_{\text {Hill }}=\frac{\mu_{V}+\mu_{R}}{2} \text { or } \sqrt{\mu_{V} \mu_{R}} \text {. }
$$

To mimic an ideal isotropic elastic solid, Hershey ${ }^{86}$ modeled the elasticity of a polycrystalline aggregate in terms of the elasticity of individual grains with a spherical boundary, and proposed the following quartic equation:

$$
\begin{aligned}
64 \mu_{H}^{4} & +16\left(4 C_{11}+5 C_{12}\right) \mu_{H}^{3} \\
& +\left[3\left(C_{11}+2 C_{12}\right)\left(5 C_{11}+4 C_{12}\right)-8\left(7 C_{11}-4 C_{12}\right) C_{44}\right] \\
& \times \mu_{H}^{2}-\left(29 C_{11}-20 C_{12}\right)\left(C_{11}+2 C_{12}\right) C_{44} \mu_{H} \\
& -3\left(C_{11}+2 C_{12}\right)^{2}\left(C_{11}-C_{12}\right) C_{44}=0 .
\end{aligned}
$$

Hershey's method, proposed independently by Kröner, ${ }^{87}$ is viewed generally as exact.

The calculated isotropic shear modulus $\mu$ of pure bcc Fe derived from the present first-principles calculations of the single-crystal elastic moduli, has been summarized in Table VI and compared with the polycrystal experimental measurements. The shear modulus $\mu$ for bcc Fe-Cu solid solution is illustrated in Fig. 10, which is calculated from the cluster expansion predicted cubic symmetry elastic constant tensor for the solid solution phase based on Eqs. (A1)-(A4). As a lower bound, the $\mu$ of Reuss' estimation becomes negative in the $\mathrm{Cu}$-rich region, which is consistent with the $C^{\prime}$ instability. Hence, the second type Hill estimation, defined as the geometrical average of the Voigt and Reuss isotropic shear modulus, has no physical meaning in that region. As for the Hershey estimation, it gives an imaginary value for $\mathrm{Cu}$ concentrations larger than about 66 at.\%.
*Present address: Department of Chemical Engineering and Materials Science, Center for Computational Science and Engineering, University of California, Davis, Davis, CA 95616.

${ }^{1}$ S. K. Lahiri and M. E. Fine, J. Met. 21, A132 (1969).

${ }^{2}$ T. Harry and D. J. Bacon, Acta Mater. 50, 195 (2002); 50, 209 (2002).

${ }^{3}$ D. J. Bacon and Yu. N. Osetsky, J. Nucl. Mater. 329-333, 1233 (2004).

${ }^{4}$ M. E. Fine and D. Isheim, Scr. Mater. 53, 115 (2005).

${ }^{5}$ E. M. Lopasso, M. Caro, A. Caro, and P. Turchi, Phys. Rev. B 68 ,
214205 (2003)

${ }^{6}$ G. M. Worrall, J. T. Buswell, C. A. English, M. G. Hetherington, and G. D. W. Smith, J. Nucl. Mater. 148, 107 (1987).

${ }^{7}$ P. J. Othen, M. L. Jenkins, G. D. W. Smith, and W. J. Phythian, Philos. Mag. Lett. 64, 383 (1991).

${ }^{8}$ W. J. Phythian, S. Dumbill, P. Brown, and R. Sinclair, Proceedings of the 6th International Symposium on Environmental Degradation in Nuclear Power Systems-Water Reactors, 1993, TMS 729

${ }^{9}$ P. J. Othen, M. L. Jenkins, and G. D. W. Smith, Philos. Mag. A 
70, 1 (1994).

${ }^{10}$ R. Monzen, M. L. Jenkins, and A. P. Sutton, Philos. Mag. A 80, 711 (2000)

${ }^{11}$ A. Deschamps, M. Militzer, and W. J. Poole, ISIJ Int. 41, 196 (2001).

${ }^{12}$ S. R. Goodman, S. S. Brenner, and J. R. Low, Jr., Metall. Trans. 4, 2363 (1973); 4, 2371 (1973).

${ }^{13}$ P. Auger, P. Pareige, S. Wetzel, and J.-C. Van Duysen, J. Nucl. Mater. 280, 331 (2000).

${ }^{14}$ D. Isheim and D. N. Seidman, Surf. Interface Anal. 36, 569 (2004).

${ }^{15}$ E. F. Kneller, J. Appl. Phys. 35, 2210 (1964).

${ }^{16}$ W. Klement, Jr., Trans. Soc. Min. Eng. AIME 233, 1180 (1965).

${ }^{17}$ K. Sumiyama, T. Yoshitake, and Y. Nakamura, J. Phys. Soc. Jpn. 53, 3160 (1984).

${ }^{18}$ C. L. Chien, S. H. Liou, D. Kofalt, W. Yu, T. Egami, and T. R. McGuire, Phys. Rev. B 33, 3247 (1986).

${ }^{19}$ K. Uenishi, K. F. Kobayashi, S. Nasu, H. Hatano, K. N. Ishihara, and P. H. Shingu, Z. Metallkd. 83, 132 (1992).

${ }^{20}$ A. R. Yavari, P. J. Desré, and T. Benameur, Phys. Rev. Lett. 68, 2235 (1992).

${ }^{21}$ J. Eckert, J. C. Holzer, and W. L. Johnson, J. Appl. Phys. 73, 131 (1993).

22 J. Eckert, J. C. Holzer, C. E. Krill, III, and W. L. Johnson, J. Appl. Phys. 73, 2794 (1993).

${ }^{23}$ E. Ma, M. Atzmon, and F. Pinkerton, J. Appl. Phys. 74, 955 (1993).

${ }^{24}$ B. Fultz, C. C. Ahn, S. Spooner, L. B. Hong, J. Eckert, and W. L. Johnson, Metall. Mater. Trans. A 27, 2934 (1996).

${ }^{25}$ L. B. Hong and B. Fultz, Acta Mater. 46, 2937 (1998).

${ }^{26}$ P. J. Schilling, J. H. He, R. C. Tittsworth, and E. Ma, Acta Mater. 47, 2525 (1999).

${ }^{27}$ P. J. Schilling, V. Palshin, R. C. Tittsworth, J. H. He, and E. Ma, Phys. Rev. B 68, 224204 (2003).

${ }^{28}$ A. F. Tatarchenko, V. S. Stepanyuk, W. Hergert, P. Rennert, R. Zeller, and P. H. Dederichs, Phys. Rev. B 57, 5213 (1998).

${ }^{29}$ J.-T. Wang, L. Zhou, Y. Kawazoe, and D.-S. Wang, Phys. Rev. B 60, 3025 (1999).

${ }^{30}$ W. Zhang and E. Ma, J. Mater. Res. 15, 653 (2000).

${ }^{31}$ C. Domain and C. S. Becquart, Phys. Rev. B 65, 024103 (2001).

${ }^{32}$ Z. Tang, M. Hasegawa, Y. Nagai, and M. Saito, Phys. Rev. B 65, 195108 (2002).

${ }^{33}$ H. R. Gong, L. T. Kong, and B. X. Liu, Phys. Rev. B 69, 054203 (2004).

${ }^{34}$ T. Kraft, P. M. Marcus, M. Methfessel, and M. Scheffler, Phys. Rev. B 48, 5886 (1993).

${ }^{35}$ P. J. Craievich, M. Weinert, J. M. Sanchez, and R. E. Watson, Phys. Rev. Lett. 72, 3076 (1994).

${ }^{36}$ L. G. Wang and M. Sob, Phys. Rev. B 60, 844 (1999).

${ }^{37}$ L. G. Wang, M. Sob, and Z. Zhang, J. Phys. Chem. Solids 64, 863 (2003)

${ }^{38}$ Z. Q. Wang, S. H. Lu, Y. S. Li, F. Jona, and P. M. Marcus, Phys. Rev. B 35, R9322 (1987).

${ }^{39}$ H. Li, D. Tian, J. Quinn, Y. S. Li, F. Jona, and P. M. Marcus, Phys. Rev. B 43, 6342 (1991).

${ }^{40}$ P. J. Craievich, J. M. Sanchez, R. E. Watson, and M. Weinert, Phys. Rev. B 55, 787 (1997).

${ }^{41}$ P. J. Craievich and J. M. Sanchez, Comput. Mater. Sci. 8, 92 (1997).

${ }^{42}$ K. Persson, M. Ekman, and G. Grimvall, Phys. Rev. B 60, 9999
(1997).

${ }^{43}$ A. Taga, L. Vitos, B. Johansson, and G. Grimvall, Phys. Rev. B 71, 014201 (2005).

${ }^{44}$ J. M. Sanchez, F. Ducastelle, and D. Gratias, Physica A 128, 334 (1984).

${ }^{45}$ A. van de Walle and M. Asta, in Handbook of Materials Modeling, edited by S. Yip (Springer, Dordrecht, The Netherlands).

${ }^{46}$ J. W. D. Connolly and A. R. Williams, Phys. Rev. B 27, R5169 (1983).

${ }^{47}$ D. de Fontaine, Solid State Phys. 47, 33 (1994).

${ }^{48}$ G. Ceder, Ph.D. thesis, University of California, 1991.

${ }^{49}$ A. van de Walle and G. Ceder, J. Phase Equilib. 23, 348 (2002).

${ }^{50}$ G. Kresse and J. Hafner, Phys. Rev. B 47, R558 (1993).

${ }^{51}$ G. Kresse and J. Hafner, J. Phys.: Condens. Matter 6, 8245 (1994)

${ }^{52}$ P. E. Blöchl, Phys. Rev. B 50, 17953 (1994).

${ }^{53}$ G. Kresse and D. Joubert, Phys. Rev. B 59, 1758 (1999).

${ }^{54}$ J. P. Perdew, J. A. Chevary, S. H. Vosko, K. A. Jackson, M. R. Pederson, D. J. Singh, and C. Fiolhais, Phys. Rev. B 46, 6671 (1992).

${ }^{55}$ H. J. Monkhorst and J. D. Pack, Phys. Rev. B 13, 5188 (1976).

${ }^{56}$ M. Methfessel and A. T. Paxton, Phys. Rev. B 40, 3616 (1989).

${ }^{57}$ J. A. Rayne and B. S. Chandrasekhar, Phys. Rev. 122, 1714 (1961).

${ }^{58}$ Y. S. Touloukian, R. K. Kirby, R. E. Taylor, and P. D. Desai, Thermophysical Properties of Matter, Thermal Expansion, Metallic Elements and Alloys (IFI/Plenum, New York, 1975), Vol. 12, p. 157.

${ }^{59}$ J. P. Perdew, K. Burke, and M. Ernzerhof, Phys. Rev. Lett. 77, 3865 (1996).

${ }^{60}$ J. P. Perdew and Y. Wang, Phys. Rev. B 45, 13244 (1992).

${ }^{61}$ D. M. Ceperley and B. J. Alder, Phys. Rev. Lett. 45, 566 (1980).

${ }^{62}$ S. H. Vosko, L. Wilk, and M. Nusair, Can. J. Phys. 58, 1200 (1980).

${ }^{63}$ E. Wigner, Phys. Rev. 46, 1002 (1934).

${ }^{64}$ Z. W. Lu, S.-H. Wei, and A. Zunger, Phys. Rev. B 41, 2699 (1990).

${ }^{65}$ L. Vocadlo, G. A. de Wijs, G. Kresse, M. Gillan, and G. D. Price, Faraday Discuss. 106, 205 (1997).

${ }^{66}$ C. Amador, W. R. L. Lambrecht, and B. Segall, Phys. Rev. B 46, R1870 (1992).

${ }^{67}$ J.-H. Cho and M. Scheffler, Phys. Rev. B 53, 10685 (1996).

${ }^{68}$ L. Stixrude, R. E. Cohen, and D. J. Singh, Phys. Rev. B 50, 6442 (1994).

${ }^{69}$ C. Elsasser, J. Zhu, S. G. Louie, M. Fahnle, and C. T. Chan, J. Phys.: Condens. Matter 10, 5081 (1998).

${ }^{70}$ G. Y. Guo and H. H. Wang, Chin. J. Phys. (Taipei) 38, 949 (2000).

${ }^{71}$ H. Ma, S. L. Qiu, and P. M. Marcus, Phys. Rev. B 66, 024113 (2002).

${ }^{72}$ Y. Wang, S. Curtarolo, C. Jiang, R. Arroyave, T. Wang, G. Ceder, L.-Q. Chen, and Z.-K. Liu, CALPHAD: Comput. Coupling Phase Diagrams Thermochem. 28, 79 (2004).

${ }^{73}$ W. C. Overton, Jr. and J. Gaffney, Phys. Rev. 98, 969 (1955).

${ }^{74}$ L. Kaufman, CALPHAD: Comput. Coupling Phase Diagrams Thermochem. 2, 117 (1978).

${ }^{75}$ S. Lee and R. Hoffmann, J. Am. Chem. Soc. 124, 4811 (2002).

${ }^{76}$ P. Asoka-Kumar, B. D. Wirth, P. A. Sterne, R. H. Howell, and G. R. Odette, Philos. Mag. Lett. 82, 609 (2002).

${ }^{77}$ J. T. Buswell, C. A. English, M. G. Hetherington, W. J. Phythian, 
G. D. W. Smith, and G. M. Worrall, Effects of Radiation on Materials: 14th International Symposium, edited by N. H. Packan, R. E. Stoller, and A. S. Kumar (American Society for Testing and Materials, Philadelphia, 1990), Vol. II, p. 127

${ }^{78}$ K. C. Russell and L. M. Brown, Acta Metall. 20, 969 (1972).

${ }^{79} \mathrm{C}$. Zener, Elasticity and Anelasticity in Metals (University of Chicago Press, Chicago, IL, 1948).

${ }^{80}$ W. J. Phythian, A. J. E. Foreman, C. A. English, J. T. Buswell, M. G. Hetherington, K. Roberts, and S. Pizzini, in Proceedings of the 15th International Symposium on the Effects of Radiation on Materials (American Society for Testing and Materials, 1992), p. 13.
${ }^{81}$ G. J. Ackland, G. I. Tichy, V. Vitek, and M. W. Finnis, Philos. Mag. A 56, 735 (1987).

${ }^{82}$ G. J. Ackland, D. J. Bacon, A. F. Calder, and T. Harry, Philos. Mag. A 75, 713 (1997).

${ }^{83}$ W. Voigt, Lehrbuch der Kristallphysik, Leipzig, Germany (Teubner, Stuttgart, 1928).

${ }^{84}$ A. Reuss, Z. Angew. Math. Mech. 9, 49 (1929).

${ }^{85}$ R. Hill, Proc. Phys. Soc., London, Sect. A 65, 349 (1952).

${ }^{86}$ A. V. Hershey, J. Appl. Mech. 21, 236 (1954).

${ }^{87}$ E. Kröner, Z. Phys. 151, 504 (1958).

${ }^{88}$ G. R. Speich, A. J. Schwoeble, and W. C. Leslie, Metall. Trans. 3, 2031 (1972). 